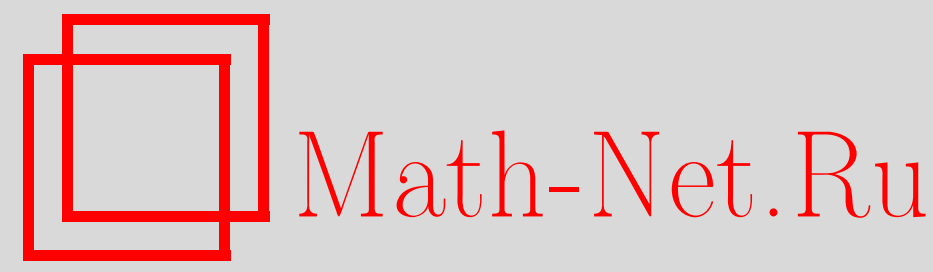

Н. А. Шананин, Об однозначном продолжении решений дифференциальных уравнений со взвешенными производными, Матем. сб., 2000, том 191, номер 3, 113-142

DOI: https://doi.org/10.4213/sm465

Использование Общероссийского математического портала Math-Net.Ru подразумевает, что вы прочитали и согласны с пользовательским соглашением http://www . mathnet.ru/rus/agreement

Параметры загрузки:

IP: 54.174 .149 .18

26 апреля 2023 г., 03:39:30 


\title{
Н.А. Шананин \\ Об однозначном продолжении решений дифференциальных уравнений со взвешенными производными
}

\begin{abstract}
Статья содержит обобщение теоремы Кальерона о локалной единственности решения задачи Коши на дифференциальные уравнения со взвешенньми производнњпи. Получены анизотропные оценки карлемановского типа. Выделен класс дифференциальных уравнений со взвешенньми производньми, ростки решений которых имеют однозначное продолжение по части переменных.

Библиография: 15 названий.
\end{abstract}

В силу теоремы Хольмгрена [1; теорема 8.6.5] для линейных дифференциальных уравнений с аналитическими коэффициентами из равенства решения однородного уравнения нулю по одну сторону от нехарактеристической $C^{1}$-гиперповерхности следует, что решение равно нулю в некоторой окрестности гиперповерхности. Для уравнений с неаналитическими, но бесконечно дифференцируемыми коэффициентами этот эффект уже не имеет места. Плись [2] построил пример эллиптического уравнения (для такого уравнения любая $C^{1}$-гиперповерхность не является характеристической), имеющего нетривиальные решения с компактным носителем. В работе Кальдерона [3] доказана теорема единственности продолжения решений уравнений через нехарактеристическую гиперповерхность для дифференциальных операторов с вешественными главными символами, характеристический многочлен которых имеет в точках гиперповерхности простые корни. Дальнейшее обобщение теоремы Кальдерона было связано с поиском достаточных условий на корни характеристического многочлена, гарантирующих однозначность продолжения. Основные результаты изложены в работах Л. Ниренберга [4], Ф. Трева [5], Л. Хёрмандера [1] и Ю.В. Егорова [6].

В настоящей статье теорема Кальдерона обобщается на дифференциальные операторы со взвешенными производными. Без учета взвешивания характеристические многочлены рассматриваемых ниже дифференциальных уравнений имеют кратные корни. Эта кратность, вообще говоря, не является постоянной. Введение взвешивания производных, с одной стороны, устраняет кратность, с другой несколько усложняет ситуацию. Вместо одного старшего символа приходится работать с пучком старших символов. Одним из основных шагов в доказательстве теоремы Кальдерона является сведение исходного линейного дифференциального уравнения к эквивалентной системе уравнений вида

$$
D_{t} \mathbf{u}+A(t) \mathbf{u}=\mathbf{f},
$$

Работа вьполнена при поддержке Российского фонда фундаментальных исследований (грант № 99-01-01139). 
где $\mathbf{u}$ и $\mathbf{f}$ - векторнозначные функции, $A(t)$ - матричнозначный псевдодифференциальный оператор. В том случае, когда производным по разным переменным в исходном уравнении присвоены различные натуральные веса, аналогичный прием приводит к системе вида $(0.1)$, в которой оператору $D_{t}$ отвечает вес $\mu \geqslant 1$, матричнозначный оператор $A(t)$ является анизотропным псевдодифференциальным оператором, имеющим полиоднородный с шагом 1 символ порядка $\mu$. Так же как в изотропном случае (все веса равны 1), выкладки достаточно проводить с точностью до операторов, ограниченных в пространстве суммируемых с квадратом функций. В связи с этим в анизотропной ситуации определенную аккуратность следует проявить в отношении учета влияния той части полного символа оператора $A(t)$, которая в асимптотическом разложении полного символа соответствует членам порядков квазиоднородности $\mu, \mu-1, \ldots, 1$. По этой причине в исходном дифференциальном уравнении выделяются влияющие на них слагаемые. Отвечающие им символы определяют пучок символов.

Теоремы о локальной единственности решений задачи Коши для линейных и квазилинейных уравнений со взвешенными производными сфформулированы в 1 . Основу их доказательства составляет оценка карлемановского типа, полученная в теореме 2.5 (см. $\S 2$ ). Доказательство теорем проводится в $\S 3$. В $\S 4$ доказаны теоремы об однозначном продолжении ростков решений, вытекающие из полученных теорем единственности. Там же теоремы о продолжении ростков решений проиллюстрированы примерами.

\section{§1. Основные результаты}

Пусть в открытом множестве $\Omega \subset \mathbb{R}^{n+1}$ определен линейньй анизотропньй дифференциальный оператор с бесконечно дифференцируемыми коэффициентами:

$$
P(x, D)=\sum_{\langle\varrho, \alpha\rangle \leqslant m} a_{\alpha}(x) D^{\alpha}, \quad D_{x_{j}}=\frac{1}{i} \frac{\partial}{\partial x_{j}}, j=1,2, \ldots, n+1,
$$

где $\alpha=\left(\alpha_{1}, \alpha_{2}, \ldots, \alpha_{n+1}\right)$ - целочисленньй мультииндекс, $\langle\varrho, \alpha\rangle-$ взвешенньй порядок производной $D^{\alpha}$ :

$$
\langle\varrho, \alpha\rangle=\varrho_{1} \alpha_{1}+\varrho_{2} \alpha_{2}+\cdots+\varrho_{n+1} \alpha_{n+1},
$$

$\varrho=\left(\varrho_{1}, \varrho_{2}, \ldots, \varrho_{n+1}\right) \in \mathbb{N}^{n+1}$. Если по крайней мере один из коэффициентов $a_{\alpha}(x)$ при $\langle\varrho, \alpha\rangle=m$ отличен от тождественного нуля, то число $m$ называется взвешенным порядком оператора.

Введем в $\mathbb{R}^{n+1}$ действие мультипликативной группы $\mathbb{R}_{+}$:

$$
\lambda \circ \xi=\left(\lambda^{\varrho_{1}} \xi_{1}, \lambda^{\varrho_{2}} \xi_{2}, \ldots, \lambda^{\varrho_{n+1}} \xi_{n+1}\right), \quad \lambda \in \mathbb{R}_{+} .
$$

Полный символ анизотропного дифференциального оператора (1.1) является суммой квазиоднородных по $\xi \in \mathbb{R}^{n+1}$ слагаемых

$$
p_{k}(x, \xi)=\sum_{\langle\varrho, \alpha\rangle=k} a_{\alpha}(x) \xi^{\alpha}
$$


степеней $k=0,1, \ldots, m$ соответственно, $(x, \xi) \in \Omega \times \mathbb{R}^{n+1}$. Символ максимальной степени $p_{m}(x, \xi)$ называется взвешенным главнылм символом оператора $P(x, D)$. Положим $\mu=\min \varrho_{j}, \nu=\max \varrho_{j}$. Оператору $P(x, D)$ поставим в соответствие пучок символов

$$
\mathscr{H}(x, \xi, h)=\sum_{k=0}^{\mu-1} h^{k} p_{m-k}(x, \xi),
$$

где $h \in \mathbb{R}$. Пучок символов $\mathscr{H}(x, \xi, h)$ является многочленом по совокупности переменных $(\xi, h)$ с бесконечно дифференцируемьми по $x$ коэффициентами и $p_{m}(x, \xi)=\mathscr{H}(x, \xi, 0)$. Определим на $\mathbb{R}$ действие группы $\mathbb{R}_{+}$, положив $\lambda \circ h=\lambda h$. Тогда пучок $\mathscr{H}(x, \xi, h)$ будет квазиоднородной функцией порядка $m$ относительно переменных $(\xi, h)$.

Обозначим через $x^{\prime}$ совокупность координат $x_{j}$, для которых вес $\varrho_{j}$ равен минимальному $\mu$, а через $x^{\prime \prime}$ совокупность координат $x_{j}$, для которых $\varrho_{j}>\mu$. Соответствующие двойственные переменные обозначаются через $\xi^{\prime}$ и $\xi^{\prime \prime}$. Гиперповерхность $S=\{x \in U: \varphi(x)=0\}$, где $U$ - область в $\Omega, \varphi(x)$ - вешественная $C^{\infty}$-функция и $d \varphi \neq 0$ на $U$, называется нехарактеристической в точке $x^{0} \in S$ для оператора $P(x, D)$, если $p_{m}\left(x^{0}, \varphi_{x^{\prime}}\left(x^{0}\right), 0\right) \neq 0$.

Пусть все точки гиперповерхности $S$ являются нехарактеристическими для оператора $P(x, D)$. Функция $p_{m}\left(x, \xi^{\prime}+z \varphi_{x^{\prime}}(x), \xi^{\prime \prime}\right)$ является многочленом по $z \in \mathbb{C}$ степени не выше $k=m / \mu$, причем коэффициент при старшей степени $k$, равный $p_{m}\left(x, \varphi_{x^{\prime}}(x), 0\right)$, отличен от нуля в некоторой окрестности $S$. Поэтому если гиперповерхность $S$ является нехарактеристической для оператора $P(x, D)$, то для любой точки $x$ из некоторой окрестности $S$ многочлен $p_{m}\left(x, \xi^{\prime}+z \varphi_{x^{\prime}}(x), \xi^{\prime \prime}\right)$ относительно $z$ имеет с учетом кратности $k$ корней. Предположим, что для оператора $P(x, D)$ и гиперповерхности $S$ выполняется условие:

(1) в точке $x^{0} \in S$ для любого вектора $\xi \in \mathbb{R}^{n+1}$, не коллинеарного $\left(\varphi_{x^{\prime}}\left(x^{0}\right), 0\right)$, многочлен $p_{m}\left(x^{0}, \xi^{\prime}+z \varphi_{x^{\prime}}\left(x^{0}\right), \xi^{\prime \prime}\right)$ от $z \in \mathbb{C}$ имеет $k$ простых корней.

Относительно переменной $z \in \mathbb{C}$ функция $\mathscr{H}\left(x, \xi+h^{\varrho-\mu} z \varphi_{x}(x), h\right)$, где $\left(\xi+h^{\varrho-\mu} z \varphi_{x}(x)\right)_{j}=\xi_{j}+h^{\varrho_{j}-\mu} z \varphi_{x_{j}}(x), j=1,2, \ldots, n+1$, является многочленом степени $k$ с бесконечно дифференцируемыми коэффициентами

$$
\mathscr{H}\left(x, \xi+h^{\varrho-\mu} z \varphi_{x}(x), h\right)=\sum_{l=0}^{k} b_{l}(x, \xi, h) z^{l}
$$

Пусть вектор $\xi^{0}$ неколлинеарен $\left(\varphi_{x^{\prime}}\left(x^{0}\right), 0\right)$. Положим $b_{l}^{0}=b_{l}\left(x^{0}, \xi^{0}, 0\right), l=$ $0,1, \ldots, k$. Тогда в силу условия (1) из комплексного варианта теоремы о неявной функции следует, что сушествует такая окрестность $W^{\prime} \subseteq \mathbb{C}^{k+1}$ точки $b^{0}=\left(b_{0}^{0}, b_{1}^{0}, \ldots, b_{k}^{0}\right) \in \mathbb{C}^{k+1}$, что при $b \in W^{\prime}$ уравнение $\sum_{l=0}^{k} b_{l} \bar{z}^{l}=0$ имеет $k$ различных корней $z_{j}^{\prime}(b), j=1,2, \ldots, k$, аналитически зависящих от $b$. Отсюда вытекает, что для каждой точки $\left(x^{0}, \xi^{0}, 0\right)$ найдется такая ее окрестность $W$, что относительно переменной $z \in \mathbb{C}$ уравнение

$$
\mathscr{H}\left(x, \xi+h^{\varrho-\mu} z \varphi_{x}(x), h\right)=0, \quad(x, \xi, h) \in W
$$


имеет $k$ различных корней $z_{j}(x, \xi, h)=z_{j}^{\prime}(b(x, \xi, h))$, бесконечно гладко зависящих от $(x, \xi, h) \in W$. Кроме того, из положительной квазиоднородности по совокупности переменных $(\xi, h)$ пучка $\mathscr{H}(x, \xi, h)$ следует, что каждый корень $z_{j}(x, \xi, h)$ является положительно квазиоднородной по $(\xi, h)$ степени $\mu$ функцией. Напомним, что множество $W$ называется квазиконическим, если из включения $(x, \xi, h) \in W$ следует $(x, \lambda \circ \xi, \lambda \circ h) \in W$ при всех $\lambda \in \mathbb{R}_{+}$. Вследствие указанной квазиоднородности можно считать, что окрестность $W$, в которой определены корни $z_{j}(x, \xi, h)$, является квазиконической.

Будем предполагать, что

(2) каждый из корней уравнения (1.2) удовлетворяет одному из условий:

(I) $\operatorname{Im} z_{j}(x, \xi, h) \equiv 0$ в $W$;

(II) $\operatorname{Im} z_{j}(x, \xi, 0) \neq 0$ в $W \cap\{h=0\}$.

Поскольку окрестность $W$ можно сужать, то для вьполнения (II) достаточно, чтобы $\operatorname{Im} z_{j}\left(x^{0}, \xi^{0}, 0\right) \neq 0$.

Для каждого целого неотрицательного числа $s$ через $H_{w}^{s}(\Omega)$ обозначим пространство функций $u \in L_{2}(\Omega)$, имеюших обобшенные производные $D^{\alpha} u \in L_{2}(\Omega)$ при $\langle\varrho, \alpha\rangle \leqslant s$. Функциональные пространства $H_{w}^{s}(\Omega)$ несушественно отличаются от известных анизотропных пространств Соболева $W_{2}^{\vec{l}}(\Omega)[7]$. Использование пространств $H_{w}^{s}(\Omega)$ в формулируемых ниже теоремах позволяет точно описывать классы априорной гладкости рассматриваемых функций.

ТЕОРема 1.1. Пусть гиперповерхность $S$ не является характеристической для $P(x, D)$ в точке $x^{0} \in S$ и выполнень условия (1) и (2). Тогда если $P(x, D) u=0$ в $U, u \in H_{w}^{m-\mu}(U)$ и $u=0$ в $U_{-}=\{x \in U: \varphi(x)<0\}$, mо $u=0$ в некоторой окрестности точки $x^{0}$.

В качестве примера рассмотрим дифференциальный оператор

$$
P=D_{x_{1}}^{3}+a(x) D_{x_{2}}^{2}+b(x) D_{x_{1}} D_{x_{2}}+c(x) D_{x_{1}}^{2}+\mathscr{L}(x, D)
$$

где $x=\left(x_{1}, x_{2}\right) \in \Omega \subset \mathbb{R}^{2}, \mathscr{L}(x, D)$ - дифференциальный оператор первого порядка. Введем взвешивание производных: $D_{x_{1}} \sim 2$ и $D_{x_{2}} \sim 3$. Тогда $\mu=2$, взвешенный главный символ $p_{6}(x, \xi)=\xi_{1}^{3}+a(x) \xi_{2}^{2}$ и пучок символов $\mathscr{H}(x, \xi, h)=p_{6}(x, \xi)+h b(x) \xi_{1} \xi_{2}$. Поверхность $S$, заданная в окрестности точки $x^{0} \in \Omega$ уравнением $\varphi(x)=0$, будет нехарактеристической в этой точке, если $\varphi_{x_{1}}\left(x^{0}\right) \neq 0$. Если $a\left(x^{0}\right) \neq 0$, то для оператора $P$ в точке $x^{0}$ выполняется условие (1). Это следует из того, что система уравнений

$$
p_{6}\left(x^{0}, \xi_{1}+z \varphi_{x_{1}}\left(x^{0}\right), \xi_{2}\right)=0, \quad \frac{\partial}{\partial z} p_{6}\left(x^{0}, \xi_{1}+z \varphi_{x_{1}}\left(x^{0}\right), \xi_{2}\right)=0
$$

совместна тогда и только тогда, когда вектор $\xi=\left(\xi_{1}, \xi_{2}\right)$ коллинеарен вектору $\left(\varphi_{x_{1}}\left(x^{0}\right), 0\right)$. Приведем два вида условий, достаточных для того, чтобы оператор удовлетворял условию (2). Если $\operatorname{Im} a\left(x^{0}\right) \neq 0$, то корни уравнения (1.2) имеют в точке $\left(x^{0}, \xi^{0}, 0\right)$ ненулевую мнимую часть. Следовательно, оператор $P$ удовлетворяет условию (2) в точке $x^{0}$. Предположим, что оба коэффициента $a(x)$ и $b(x)$ являются вешественнозначными функциями и $a\left(x^{0}\right) \neq 0$. Тогда функция $\mathscr{H}(x, \xi, h)$ 
при вещественных $(x, \xi, h)$ принимает вещественные значения. В силу вещественного варианта теоремы о неявной функции один из корней уравнения (1.2) удовлетворяет условию (I). Два других удовлетворяют условию (II). Следовательно, в этом случае для оператора $P$ в точке $x^{0}$ выполняется условие (2). Предположим, что оператор удовлетворяет условию (2). В силу теоремы 1.1 решения уравнения $P u=f$ обладают следуюшим свойством единственности продолжения через нехарактеристическую поверхность. Пусть $u \in \mathscr{D}^{\prime}(\Omega), u, u_{x_{1}}, u_{x_{2}}, u_{x_{1}, x_{1}} \in L_{2}(U)$, $P(x, D) u=0$ в $U$ и $u=0$ в $U_{-}$. Тогда $u=0$ в некоторой окрестности точки $x^{0}$.

Теорема 1.1 применима к анизотропньм строго гиперболическим дифференциальным операторам. Предположим, что взвешенные символы $p_{m}(x, \xi), p_{m-1}(x, \xi)$, $\ldots, p_{m-\mu+1}(x, \xi)$ вешественнозначны. Тогда пучок $\mathscr{H}(x, \xi, h)$ при вешественных $x, \xi$ и $h$ принимает вешественные значения. Назовем оператор $P$ обобщенно строго гиперболическим в точке $x^{0}$ относительно гиперповерхности $S, x^{0} \in S$, если $p_{m}\left(x^{0}, \varphi_{x^{\prime}}\left(x^{0}\right), 0\right) \neq 0$ и для любого вектора $\xi$, не коллинеарного вектору $\left(\varphi_{x^{\prime}}\left(x^{0}\right), 0\right)$, уравнение $p_{m}\left(x^{0}, \xi^{\prime}+z \varphi_{x^{\prime}}\left(x^{0}\right), \xi^{\prime \prime}\right)=0$ относительно $z$ имеет $k$ различных вещественных корней. В этом случае из вешественного варианта теоремы о неявной функции следует, что корни уравнения (1.2) удовлетворяют условию (I).

Теорема 1.1 имеет приложение к задачам с краевым условием типа Кошт. Пусть точка $x^{0} \in \partial \Omega, u \in \mathscr{D}^{\prime}(\Omega)$ и $s$ - целое неотрицательное число. Будем говорить, что $u \in \dot{H}_{w}^{s}\left(x^{0}\right)$, если сушествует окрестность $U$ точки $x^{0}$ такая, что для любой функции $\varphi(x) \in \mathscr{D}(U)$ произведение $\varphi$ п принадлежит пополнению пространства $\mathscr{D}(U \cap \Omega)$ относительно нормы

$$
\left(\sum_{\langle\varrho, \alpha\rangle \leqslant s} \int_{U \cap \Omega}\left|D^{\alpha} u\right|^{2} d x\right)^{1 / 2} .
$$

Поясним включение $u \in \dot{H}_{w}^{s}\left(x^{0}\right)$. Если $\partial \Omega \in C^{\infty}$ в окрестности $U$ и $\partial \Omega$ трансверсальна слою $\left\{x: x^{\prime \prime}=x^{\prime \prime 0}\right\}$ в точке $x^{0}$, то найдется такая окрестность $V \subset U$ точки $x^{0}$, что при $\langle\varrho, \alpha\rangle+\mu \leqslant s$ для функции $u \in H_{w}^{s}$ определены сужения производных $\left.D^{\alpha} u\right|_{V \cap \partial \Omega} \in L_{2}(V \cap \partial \Omega)$. Если $u \in \dot{H}_{w}^{s}\left(x^{0}\right)$, то $\left.D^{\alpha} u\right|_{\partial \Omega}=0$.

Предположим, что оператор $P(x, D)$ определен на множестве $\Omega \cup U$.

ТЕОРема 1.2. Пусть через точку $x^{0} \in \partial \Omega$ можсно провести нехарактеристическую в $x^{0}$ гиперповерхность $S=\{x \in U: \varphi(x)=0\}$ такую, что $\varphi(x)>0$ при $x \in U \cap \Omega$. Пусть, кроме того, для $P(x, D)$ в точке $x^{0}$ выполняются условия (1) и (2). Тогда если $u \in \dot{H}_{w}^{m}\left(x^{0}\right)$ и $P(x, D) u=0$ в $U \cap \Omega$, то и некоторой окрестности точки $x^{0}$.

Таким образом, если в точке $x^{0} \in \partial \Omega$ выполнены условия теоремы 1.2 относительно $P(x, D)$ и $S$, то решения $u_{1}$ и $u_{2}$ уравнения $P(x, D) u=f$ в $\Omega$, различающиеся на функцию класса $\dot{H}_{w}^{m}\left(x^{0}\right)$, совпадают в некоторой окрестности $x^{0}$.

Теорему 1.1 можно распространить на квазилинейные дифференциальные уравнения вида

$$
P(x, D) u=F\left(x, D^{\beta} u\right), \quad\langle\varrho, \beta\rangle \leqslant m-\mu .
$$

Уравнение понимается в следуюшем смысле. Налагаемые на $F$ и $u$ условия априорной гладкости обеспечивают принадлежность правой части уравнения пространству непрерывных функций. Левая часть есть результат действия дифференциального оператора на обобшенную функцию $u \in \mathscr{D}^{\prime}(\Omega)$. 
Пусть $N_{m-\mu}$ - количество неотрицательных целочисленных мультииндексов $\beta$ таких, что $\langle\varrho, \beta\rangle \leqslant m-\mu ; F: \Omega \times \mathbb{C}^{N_{m-\mu}} \rightarrow \mathbb{C}$ - непрерывное отображение, удовлетворяющее условию:

(3) для любых компактного множества $K \subset \Omega$ и ограниченного множества $B \subset \mathbb{C}^{N_{m-\mu}}$ сушествует константа $C(K, B)$, с которой для всех $x \in K$ и $\zeta^{1}, \zeta^{2} \in B$ справедлива липшицева оценка

$$
\left|F\left(x, \zeta^{1}\right)-F\left(x, \zeta^{2}\right)\right| \leqslant C(K, B)\left|\zeta^{1}-\zeta^{2}\right| .
$$

Для каждого целого неотрицательного числа $s$ через $C_{w}^{s}(\Omega)$ обозначим пространство функций $u$, непрерывных на $\Omega$ вместе со всеми своими производными $D^{\alpha} u$ порядка $\langle\varrho, \alpha\rangle \leqslant s$.

ТЕОРема 1.3. Предположим, что в точке $x^{0}$ поверхность $S$ не является характеристической для оператора $P(x, D)$, оператор $P(x, D)$ в точке $x^{0}$ удовлетворяет условиям (1) $и$ (2) и для непрерывного отображсения $F: \Omega \times$ $\mathbb{C}^{N_{m-\mu}} \rightarrow \mathbb{C}$ вьгполняется условие (3). Тогда если $u_{1}, u_{2} \in C_{w}^{m-\mu}(\Omega)-$ два решения уравнения

$$
P(x, D) u=F\left(x, D^{\beta} u\right), \quad x \in \Omega \quad\left(\zeta_{\beta}=D^{\beta} u\right),
$$

и $u_{1}=u_{2}$ в $U_{-}$, то $u_{1}=u_{2}$ в некоторой окрестности точки $x^{0}$.

Если предположить бо́льшую гладкость решений, то теорему 1.3 можно усилить.

Теорема 1.4. Предположим, что выполнень условия теоремы 1.3. Тогда если $u_{1}, u_{2} \in C_{w}^{m}(\Omega)$ - два решения уравнения (1.3) и $\left.D^{\alpha}\left(u_{1}-u_{2}\right)\right|_{S}=0$ при $\langle\varrho, \alpha\rangle \leqslant m-\mu$, то $u_{1}=u_{2}$ в некоторой окрестности точки $x^{0}$.

Имеет место аналог теоремы 28.1.1 из монографии Л. Хёрмандера [1] для операторов со взвешенными производными. Предположим, что

(4) множество

$$
\left\{(x, \xi, h) \in \Omega \times\left(\mathbb{R}^{n+1} \backslash\{0\}\right) \times \mathbb{R}: \mathscr{H}(x, \xi, h)=0\right\}
$$

в окрестности каждой принадлежащей ему точки $\left(x^{0}, \xi^{0}, 0\right)$ является $C^{\infty}$ гиперповерхностью.

Обозначим через $\Xi$ множество точек $\left(x, \eta^{\prime}\right) \in \Omega \times\left(\mathbb{R}^{n_{1}} \backslash\{0\}\right)$, для которых найдется такой вектор $\xi \in \mathbb{R}^{n+1}$, что полином $p_{m}\left(x, \xi^{\prime}+z \eta^{\prime}, \xi^{\prime \prime}\right)$ по $z \in \mathbb{C}$ имеет по крайней мере один корень $z^{0}$ кратности выше единицы и $\xi+z^{0} \eta \neq 0, n_{1}-$ число переменных минимального веса $\mu$.

Теорема 1.5. Пусть поверхность $S=\{x \in U: \varphi(x)=0\}$, где $U \subset \Omega, \varphi(x)$ - вещественная $C^{1}$-функиия $u \varphi_{x} \neq 0$ на $U$, не является характеристической для $P(x, D)$ в точке $x^{0}$ и выполнено условие $(4)$. Тогда если $\left(x^{0}, \varphi_{x^{\prime}}\left(x^{0}\right)\right) \notin \Xi$, $u \in H_{w}^{m-\mu}(U), P(x, D) u \in L_{2}(U), u=0$ в $U_{-}$и для любого компактного подмнохества $K \subset U$ с некоторой константой $C(K)$ для почти всех $x \in K$ справедливо неравенство

$$
|P(x, D) u| \leqslant C(K) \sum_{\langle\varrho, \alpha\rangle \leqslant m-\mu}\left|D^{\alpha} u\right|,
$$

то и 0 в некоторой окрестности точки $x^{0}$. 


\section{§2. Оценки карлемановского типа}

Основным результатом этого параграффа является доказательство оценок карлемановского типа для анизотропного дифференциального оператора

$$
P=D_{t}^{k}+\sum_{j=0}^{k-1} \sum_{\langle\varrho, \alpha\rangle \leqslant m-\mu j} a_{\alpha, j}(x, t) D_{x}^{\alpha} D_{t}^{j}
$$

где $\mu k=m, x \in \mathbb{R}^{n}, t \in\left(-T_{0}, T_{0}\right)$. Здесь для удобства выделена переменная $t$, которой отвечает вес $\mu$. В обозначениях $\S 1$ полная совокупность координат состоит из $(x, t)=\left(x_{1}, \ldots, x_{n}, t\right)$. Координате $x_{j}$ соответствует вес $\varrho_{j} \geqslant \mu, j=1,2, \ldots, n$.

Предположим, что пучок символов $\mathscr{H}_{P}(x, t, \xi, \tau, h)$ оператора $(2.1)$ удовлетворяет условию:

$\left(1^{\prime}\right)$ для любого вектора $\xi^{0} \neq 0$ уравнение $\mathscr{H}_{P}\left(0,0, \xi^{0}, \tau, 0\right)=0$ имеет $k$ простых корней по $\tau$.

Тогда в силу теоремы о неявной функции в некоторой квазиконической по $(\xi, h)$ окрестности $W$ точки $\left(0,0, \xi^{0}, 0\right)$ уравнение $\mathscr{H}_{P}(x, t, \xi, \tau, h)=0$ относительно $\tau$ имеет $k$ решений $\tau_{j}(x, t, \xi, h), j=1,2, \ldots, k$. Предположим, что

$\left(2^{\prime}\right)$ каждый корень уравнения удовлетворяет одному из условий:

$\left(\mathrm{I}^{\prime}\right)$ для любого $l=0,1, \ldots, \mu-1$

$$
\operatorname{Im} \frac{\partial^{l} \tau_{j}}{\partial h^{l}}(x, t, \xi, 0) \equiv 0
$$

на $W \cap\{h=0\}$;

$$
\left(\mathrm{II}^{\prime}\right) \operatorname{Im} \tau_{j}(x, t, \xi, 0) \neq 0 \text { на } W \cap\{h=0\} .
$$

Мы хотим показать, что при выполнении этих условий для оператора $P$ в достаточно малой окрестности начала координат $U \times(-T, T)$ для всех функций $u \in H_{w}^{m-\mu}(U \times(-T, T))$ с $P u \in L_{2}(U \times(-T, T))$ и носителем, содержащимся в $U \times(-T, T)$, с некоторой константой $C>0$ при $\rho \geqslant \rho_{*}(T)$ выполняется оценка

$$
\rho \sum_{\langle\varrho, \alpha\rangle \leqslant m-\mu} \int_{-T}^{T}\left\|D_{x, t}^{\alpha} u\right\|^{2} e^{2 \rho \phi(t)} d t \leqslant C \int_{-T}^{T}\|P u\|^{2} e^{2 \rho \phi(t)} d t
$$

где $\phi(t)=\frac{1}{2} t^{2}-2 t T$ и $\rho_{*}(T)-$ некоторое число, зависящее от $T$.

Общая схема доказательства этой оценки аналогична известньм. Первый шаг восходит к А.П. Кальдерону и состоит в замене скалярного уравнения $P u=f$ системой дифференциальных уравнений по $t$ первого порядка. Полученная при этом система является псевдодифференциальной по $x$. Затем с помощью исчисления псевдодифференциальных операторов проводится диагонализация системы, и дело сводится к доказательству оценок для конечного числа скалярных дифференциальных по $t$ уравнений первого порядка. Отличие от изотропного случая связано с тем, что в ходе проводимых выкладок наряду с взвешенным главным символом необходимо учитывать часть младших. Это есть следствие того, что взвешенњый 
порядок оператора $D_{t}$ может быть больше единицы, в то время как шаг в степенях разложения полного символа исходного оператора в сумму квазиоднородных слагаемых равен единице.

Анизотропные псевдодифференциальные операторы. В работах Р. Ласкара [8] и Т. Сакураи [9] операторы этого класса использовались при изучении распространения особенностей решений анизотропных уравнений. В работе Х. Кумано-го [10; гл. 7] построено исчисление анизотропных псевдодифференциальных операторов. Для полноты изложения ниже приведены используемые в доказательствах факты теории анизотропных псевдодифференциальных операторов.

Положим

$$
|\xi|_{\varrho}=\left(\sum_{j=1}^{n} \xi_{j}^{M_{j}}\right)^{1 / M}, \quad M=2 \prod_{j=1}^{n} \varrho_{j}, \quad M_{j}=\frac{M}{\varrho_{j}}
$$

Функция $|\xi|_{\varrho}$ положительно квазиоднородна степени единица, отлична от нуля при ненулевых $\xi$ и удовлетворяет неравенству треугольника. Пусть $m \in \mathbb{R}$. Будем говорить, что функция $a(x, \xi) \in C^{\infty}\left(\mathbb{R}^{n} \times \mathbb{R}^{n}\right)$ принадлежит классу символов $S_{w}^{m}$, если для любых неотрицательных целочисленных мультииндексов $\alpha$ и $\beta$ найдутся константы $C_{\alpha, \beta}$, с которыми для всех $(x, \xi) \in \mathbb{R}^{n} \times \mathbb{R}^{n}$ выполняются оценки

$$
\left|a_{(\beta)}^{(\alpha)}(x, \xi)\right| \leqslant C_{\alpha, \beta}\left(1+|\xi|_{\varrho}\right)^{m-\langle\varrho, \alpha\rangle},
$$

где $a_{(\beta)}^{(\alpha)}(x, \xi)=\partial_{\xi}^{\alpha} \partial_{x}^{\beta} a(x, \xi)$. Очевидно, функция $\Lambda(\xi)=\left(1+|\xi|_{\varrho}^{M}\right)^{1 / M} \in S_{w}^{1}$ является весовой в смысле определения 1.1 из [10; гл. 7]. Порожденный ею класс символов $S_{\Lambda, 1,0}^{m}$ содержит класс $S_{w}^{m}$. Отметим, что если $C^{\infty}$-функция $a(x, \xi)$ на $\mathbb{R}^{n} \times\left(\mathbb{R}^{n} \backslash\{0\}\right)$ является положительно квазиоднородной степени $m$ по $\xi$, а функция $\chi(\xi) \in \mathscr{D}\left(\mathbb{R}^{n}\right)$ равна единице в окрестности начала координат, то $(1-\chi) a \in S_{w}^{m}$.

Пусть $a_{j} \in S_{w}^{m_{j}}, j=1,2, \ldots, m_{1}>m_{2}>\cdots \rightarrow-\infty$ и $a \in C^{\infty}\left(\mathbb{R}^{n} \times \mathbb{R}^{n}\right)$. Будем писать

$$
a \sim \sum_{j=1}^{\infty} a_{j}
$$

если для любого $k>1$

$$
a-\sum_{j<k} a_{j} \in S_{w}^{m_{k}}
$$

Допуская некоторую вольность, говорят, что $a \in S_{w}^{m}$ асимптотически эквивалентен ряду из положительно квазиоднородных $C^{\infty}$-функций $a_{j}$ монотонно убывающих степеней $m_{j} \rightarrow-\infty$ при $j \rightarrow \infty$, если $a \sim \sum_{j=0}^{\infty}(1-\chi) a_{j}$. Применяя аналог предложения 18.1.4 из [1], несложно доказать, что для любого целого числа $k$ справедливо асимптотическое разложение

$$
\Lambda^{k}(\xi) \sim|\xi|_{\varrho}^{k}+\frac{k}{M}|\xi|_{\varrho}^{k-M}+\cdots, \quad M=2 \prod_{j=1}^{n} \varrho_{j} \geqslant 2 \mu
$$


Символ назьвается квазиполиоднородным, если он асимптотически эквивалентен ряду из положительно квазиоднородных функций целых монотонно убывающих степеней. Символ старшей степени квазиоднородности называется главным.

Символу $a \in S_{w}^{m}$ соответствует псевдодифференциальный оператор

$$
a(x, D) u=\left(\frac{1}{2 \pi}\right)^{n} \int e^{i\langle x, \xi\rangle} a(x, \xi) \widetilde{u}(\xi) d \xi
$$

где $\widetilde{u}(\xi)$ - преобразование Фурье функции $u(x)$ из пространства Шварца $S\left(\mathbb{R}^{n}\right)$, $\langle x, \xi\rangle=x_{1} \xi_{1}+x_{2} \xi_{2}+\cdots+x_{n} \xi_{n}$.

Определим пространство $H^{s}, s \in \mathbb{R}$, как пространство всех функций $u \in S^{\prime}\left(\mathbb{R}^{n}\right)$,

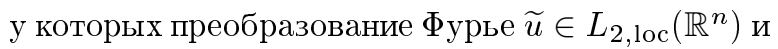

$$
\|u\|_{s}=\left(\int\left(1+|\xi|_{\varrho}\right)^{2 s}|\widetilde{u}(\xi)|^{2} d \xi\right)^{1 / 2}<\infty .
$$

Следуюшая теорема есть вариант хорошо известных теорем.

Tеорема 2.1. Пусть $a_{1} \in S_{w}^{m_{1}}, a_{2} \in S_{w}^{m_{2}}$. Тогда

1) оператор $a_{1}(x, D)$ непрерывно отображсает пространство $S\left(\mathbb{R}^{n}\right)$ в себя;

2) композичия операторов $a_{1}(x, D) \circ a_{2}(x, D)$ является псевдодифференииальным оператором с символом $a \in S_{w}^{m_{1}+m_{2}}$, причем

$$
a(x, \xi) \sim \sum_{\alpha} \frac{(-i)^{|\alpha|}}{\alpha !} a_{1}^{(\alpha)}(x, \xi) a_{2,(\alpha)}(x, \xi)
$$

əде $|\alpha|=\alpha_{1}+\alpha_{2}+\cdots+\alpha_{n} ;$

3) сопряхсенный оператор $a_{1}^{*}(x, D)$ является псевдодифференциальным оператором с символом $b \in S_{w}^{m_{1}}$, причем

$$
b(x, \xi) \sim \sum_{\alpha} \frac{(-i)^{|\alpha|}}{\alpha !} \bar{a}_{1,(\alpha)}^{(\alpha)}(x, \xi)
$$

4) для любого $s \in \mathbb{R}$ оператор а $(x, D)$ ограничен из $H^{s}$ в $H^{s-m}$.

Доказательство близких утверждений можно найти в [10]. Небольшое отличие от доказанных там утверждений связано с тем, что класс символов $S_{\Lambda, 1,0}^{m}$ шире класса $S_{w}^{m}$. Несложный анализ обоснованных в [10] асимптотических разложений символов позволяет легко устранить это различие.

Ниже теорема 2.1 будет применяться к однопараметрическим семействам символов. Предположим, что $a(t, x, \xi) \in S_{w}^{m}$ для всех $t \in\left(-T_{0}, T_{0}\right)$ и в оценках $(2.3)$ коэффициенты $C_{\alpha, \beta}$ могут быть выбраны не зависяшими от $t$. Тогда семейство операторов $a(t, x, D), t \in\left(-T_{0}, T_{0}\right)$, равностепенно непрерьвно из $H^{s}$ в $H^{s-m}$ [10; гл. 7, теорема 1.6].

Матричнозначный квазиполиоднородный символ

$$
A(x, \xi)=\left(a_{i, j}(x, \xi)\right)_{i, j=1}^{k} \in S_{w}^{m} \otimes\left(\mathbb{C}^{k} \otimes \mathbb{C}^{k}\right)
$$


называется квазиэллиптическим в точке $\left(x^{0}, \xi^{0}\right)$, если определитель главного символа $\operatorname{det} A_{m}\left(x^{0}, \xi^{0}\right)$ отличен от нуля.

При диагонализации системы уравнений окажется полезным следующий факт о квазиэллиптическом продолжении символа. Пусть в квазиконической окрестности $W$ точки $\left(x^{0}, \xi^{0}\right) \in \mathbb{R}^{n} \times\left(\mathbb{R}^{n} \backslash\{0\}\right)$ определены матричнозначные квазиоднородные по $\xi C^{\infty}$-функции $A_{m-j}(x, \xi)$ степеней $m-j$ соответственно, $j=0,1,2, \ldots, l$, причем $A_{m}$ является квазиэллиптической в $W$. Тогда существуют квазиполиоднородный символ $\widetilde{A}(x, \xi) \in S_{w}^{m} \otimes\left(\mathbb{C}^{k} \otimes \mathbb{C}^{k}\right)$, квазиконическая окрестность $W^{\prime} \subset W$ точки $\left(x^{0}, \xi^{0}\right)$ и числа $d>0$ и $\delta>0$ такие, что

$$
\widetilde{A}(x, \xi)=\sum_{j=0}^{l} A_{m-j}(x, \xi)
$$

на $W^{\prime} \cap\left\{|\xi|_{\varrho}>d\right\}$ и при всех $(x, \xi) \in \mathbb{R}^{n} \times\left(\mathbb{R}^{n} \backslash\{0\}\right)$ для главного символа вьполняется оценка

$$
\operatorname{det} \widetilde{A}_{m}(x, \xi) \geqslant \delta|\xi|_{\varrho}^{m k}
$$

Доказательство утверждения можно провести по хорошо известной схеме (см., например, статью Ниренберга [4; $§ 7]$ ). Небольшое отличие состоит в том, что продолжение символа строится по всем координатам и вместо однородных конструкций следует использовать квазиоднородные.

Диагонализация. Пусть $u \in C_{0}^{\infty}\left(\mathbb{R}^{n} \times\left(-T_{0}, T_{0}\right)\right)$. Положим

$$
v^{j}=\Lambda^{m-\mu j}(D) D_{t}^{j-1} u, \quad j=1, \ldots, k,
$$

где $\Lambda(D)$ - анизотропный псевдодифференциальный оператор с символом $\Lambda(\xi) \in$ $S_{w}^{\mu}$. Очевидно, функции $v^{j}$ принадлежат пространству $C_{0}^{\infty}\left(\left(-T_{0}, T_{0}\right) ; S\left(\mathbb{R}^{n}\right)\right)$, состоящему из финитных бесконечно дифференцируемых функций переменной $t \in\left(-T_{0}, T_{0}\right)$ со значениями в пространстве Шварца $S\left(\mathbb{R}^{n}\right)$. При этом если скалярная функция $u$ удовлетворяет уравнению $P u=f$, то функции $v^{j}$ удовлетворяют системе

$$
\left\{\begin{array}{l}
D_{t} v^{j}-\Lambda(D)^{\mu} v^{j+1}=0, \quad j \leqslant k-1 \\
D_{t} v^{k}+\sum_{j=1}^{k} q_{j}\left(x, t, D_{x}\right) v^{j}=f
\end{array}\right.
$$

где $q_{j}\left(x, t, D_{x}\right)$ - анизотропный псевдодифференциальный по $x$ оператор с символом

$$
q_{j}(x, t, \xi)=\sum_{\langle\varrho, \alpha\rangle \leqslant m-\mu(j-1)} a_{\alpha, j-1}(x, t) \xi^{\alpha} \Lambda^{-m+\mu j}(\xi) .
$$

Символ $q_{j}(x, t, \xi)$ при каждом $t \in\left(-T_{0}, T_{0}\right)$ является квазиполиоднородным символом из пространства $S_{w}^{\mu}$, бесконечно гладко зависящим от $t$.

В матричной форме система (2.4) имеет вид

$$
\left(I D_{t}+A\left(x, t, D_{x}\right)\right) \mathbf{v}=\mathbf{f}
$$


где $I$ - единичная $(k \times k)$-матрица, $\mathbf{v}$ и $\mathbf{f}$ - соответствующие вектор-столбцы. Матричный пучок символов $\mathscr{H}_{A}(x, t, \xi, h)$ матричного оператора $A\left(x, t, D_{x}\right)$ имеет вид

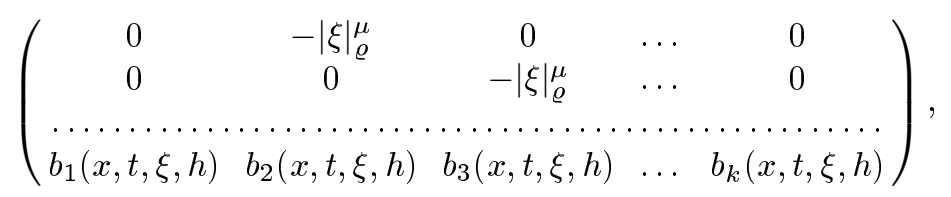

где

$$
b_{l}(x, t, \xi, h)=\sum_{j=0}^{\mu-1} h^{j} \sum_{\langle\varrho, \alpha\rangle=m-\mu(l-1)-j} a_{\alpha, l-1}(x, t) \xi^{\alpha}|\xi|_{\varrho}^{\mu l-m}, \quad l=1,2, \ldots, k .
$$

Легко видеть, что

$$
\operatorname{det}\left(\tau I+\mathscr{H}_{A}(x, t, \xi, h)\right)=\mathscr{H}_{P}(x, t, \xi, \tau, h) .
$$

В силу условия $\left(1^{\prime}\right)$ для любого $\xi^{0} \neq 0$ сушествует квазиконическая по $(\xi, h)$ окрестность $W$ точки $\left(0,0, \xi^{0}, 0\right)$, в которой задача на собственные значения

$$
\left(\tau I+\mathscr{H}_{A}(x, t, \xi, h)\right) \mathbf{e}=0
$$

имеет ровно $k$ линейно независимых решений $\mathbf{e}^{j}(x, t, \xi, h)$, соответствуюших корням $\tau_{j}(x, t, \xi, h)$. Из квазиоднородности следует, что эти решения могут быть выбраны квазиоднородными порядка нуль.

Для матричнозначной функции $\mathscr{E}(x, t, \xi, h)$, составленной из вектор-столбцов $\mathbf{e}^{j}$, в $W$ выполняется равенство

$$
\mathscr{H}_{A} \mathscr{E}=\mathscr{E} \operatorname{diag}\left(-\tau_{1}, \ldots,-\tau_{k}\right)
$$

Заметим, что производная $\frac{\partial^{l} g}{\partial h^{l}}(x, t, \xi, 0)$ бесконечно дифференцируемой в $W$ функции $g(x, t, \xi, h)$, квазиоднородной по $(\xi, h)$ порядка $s$, является бесконечно дифференцируемой функцией в $W^{\prime}=W \cap\{h=0\}$, квазиоднородной по $\xi$ порядка $s-l$. Поэтому, дифференцируя по $h$ и полагая затем $h=0$, можно построить в окрестности $W^{\prime}$ точки $\left(0,0, \xi^{0}\right)$ квазиоднородные по $\xi$ скалярные и матричные функции

$$
\varkappa_{\mu-l}^{j}(x, t, \xi)=\frac{\partial^{l} \tau_{j}}{\partial h^{l}}(x, t, \xi, 0), \quad E_{-l}(x, t, \xi)=\frac{\partial^{l} \mathscr{E}}{\partial h^{l}}(x, t, \xi, 0)
$$

степеней квазиоднородности $\mu-l$ и $-l$ соответственно, $l=0,1, \ldots, \mu-1$. При этом в силу условия $\left(2^{\prime}\right)$ в возможно суженной окрестности $W^{\prime}$ точки $\left(0,0, \xi^{0}\right)$ для каждого $j$ имеет место одно из свойств:

(i) $\operatorname{Im} \varkappa_{\mu-l}^{j}(x, t, \xi) \equiv 0$ при $l=0,1, \ldots, \mu-1$;

(ii) $\operatorname{Im} \varkappa_{\mu}^{j}(x, t, \xi) \geqslant \delta|\xi|_{\varrho}^{\mu}$, где $\delta>0$. 
Пусть $\sum_{j} A_{\mu-j}$ - разложение полного символа оператора $A\left(x, t, D_{x}\right)$ в асимптотический ряд по квазиоднородным функциям. Тогда вследствие (2.6) выражение

$$
\sum_{l=0}^{\mu-1} A_{\mu-l} \sum_{j=0}^{\mu-1} E_{-j}+\sum_{j=0}^{\mu-1} E_{-j} \operatorname{diag}\left(\sum_{l=0}^{\mu-1} \varkappa_{\mu-l}^{1}, \ldots, \sum_{l=0}^{\mu-1} \varkappa_{\mu-l}^{k}\right)
$$

является суммой конечного числа квазиоднородных слагаемых неположительных порядков.

Сужая окрестность $W^{\prime}$, можно считать, что она имеет вид $W^{\prime}=U \times\left(-T_{1}, T_{1}\right) \times \Gamma$, где $U$ - окрестность начала координат в $\mathbb{R}^{n}, T_{1}>0$ и $\Gamma$ - квазиконическая окрестность точки $\xi^{0}$. Отправляясь от сумм $\sum_{l=0}^{\mu-1} \varkappa_{\mu-l}^{j}(x, t, \xi)$ и $\sum_{l=0}^{\mu-1} E_{-l}(x, t, \xi)$, построим продолженные на $\Pi=\mathbb{R}_{x}^{n} \times\left(-T_{1}, T_{1}\right) \times \mathbb{R}_{\xi}^{n}$ символы $\varkappa^{\prime j}(x, t, \xi) \in S_{w}^{\mu}$ и $E^{\prime}(x, t, \xi) \in S_{w}^{0} \otimes\left(\mathbb{C}^{k} \otimes \mathbb{C}^{k}\right)$, бесконечно гладко зависящие от $t \in\left(-T_{1}, T_{1}\right)$, так, что для некоторого числа $d>0$ в уменњшенной окрестности $U^{\prime} \times\left(-T_{1}^{\prime}, T_{1}^{\prime}\right) \times \Gamma^{\prime}$ точки $\left(0,0, \xi^{0}\right)$ при $|\xi|_{\varrho} \geqslant d$ справедливы равенства

$$
\varkappa^{\prime j}=\sum_{l=0}^{\mu-1} \varkappa_{\mu-l}^{j}(x, t, \xi), \quad E^{\prime}=\sum_{l=0}^{\mu-1} E_{-l}(x, t, \xi) .
$$

Продолжение символов (способ указан в $[4 ; \S 7])$ можно осуществить так, что продолженные символы будут квазиполиоднородньми и, кроме того, будут удовлетворять следующим требованиям. Символ $E^{\prime}$ является квазиэллиптическим в П, и для его главного взвешенного символа при некотором числе $\delta_{1}>0$ имеет место оценка

$$
\left|\operatorname{det} E_{0}^{\prime}\right| \geqslant \delta_{1}, \quad(x, t, \xi) \in \Pi, \quad \xi \neq 0 .
$$

Символы $\varkappa^{\prime}, j=1,2, \ldots, k$, в зависимости от того, какому из условий (i) или (ii) удовлетворяет исходная сумма $\sum_{l=0}^{\mu-1} \varkappa_{\mu-l}^{j}(x, t, \xi)$, обладают одним из свойств:

(i') $\operatorname{Im} \varkappa_{\mu-l}^{\prime j} \equiv 0$ в П при $l=0,1, \ldots, \mu-1$;

$\left(\right.$ ii $\left.^{\prime}\right)$ при некотором числе $d>0$ в П выполняется оценка

$$
\left|\operatorname{Im} \varkappa_{\mu}^{\prime j}\right| \geqslant d|\xi|_{\varrho}^{\mu} .
$$

В ходе дальнейших рассуждений участвуют только семейства операторов $\varkappa^{\prime}(x, t, D)$ и $E^{\prime}(x, t, D)$. В целях упрощения записи ниже опускаются штрихи в обозначениях продолженных семейств символов $\varkappa^{\prime j}$ и $E^{\prime}$, в обозначениях соответствующих им семейств операторов $\varkappa^{\prime j}(x, t, D)$ и $E^{\prime}(x, t, D)$, а также в обозначениях задающих окрестность точки $\left(0,0, \xi^{0}\right)$ числа $T_{1}^{\prime}$ и окрестностей $U^{\prime}$ и $\Gamma^{\prime}$. Итак, если оператор $P$ вида (2.1) удовлетворяет условиям $\left(1^{\prime}\right)$ и $\left(2^{\prime}\right)$, то для любой точки $\xi^{0} \neq 0$ найдутся положительное число $T_{1}$, окрестность $U$ начала координат в $\mathbb{R}^{n}$ и квазиконическая окрестность $Г$ точки $\xi^{0}$ такие, что выполняются следующие условия: каждый из символов $\varkappa^{j}$ удовлетворяет одному из условий $\left(\mathrm{i}^{\prime}\right)$ или (ii $\left.{ }^{\prime}\right)$; взвешенный главный символ $E_{0}$ оператора $E(x, t, D)$ удовлетворяет $(2.9)$; вследствие $(2.8)$ для любого $\psi=\psi(x, \xi) \in S_{w}^{0}$ с носителем в $U \times \Gamma$ построенное семейство символов $\left(A \cdot E+E \cdot \operatorname{diag}\left(\varkappa^{1}, \varkappa^{2}, \ldots, \varkappa^{k}\right)\right) \in S_{w}^{\mu} \otimes\left(\mathbb{C}^{k} \otimes \mathbb{C}^{k}\right)$ при всех $t \in\left(-T_{1}, T_{1}\right)$ удовлетворяет включению

$$
\psi\left(A \cdot E+E \cdot \operatorname{diag}\left(\varkappa^{1}, \varkappa^{2}, \ldots, \varkappa^{k}\right)\right) \in S_{w}^{0} \otimes\left(\mathbb{C}^{k} \otimes \mathbb{C}^{k}\right) .
$$




\section{Вспомогательные оценки для скалярных операторов.}

Лемма 2.2. Пусть символ оператора $\varkappa\left(x, t, D_{x}\right)$ принадлежит $S_{w}^{\mu}$ и удовлетворяет одному из условий $\left(\mathrm{i}^{\prime}\right)$ или (ii' $\left.{ }^{\prime}\right)$. Тогда существуют числа $\delta_{2} \in$ $\left(0, T_{1}\right)$ и $\rho_{*}$ такие, что для всех $T \in\left(0, \delta_{2}\right]$ и функиий $v \in C_{0}^{\infty}\left((-T, T) ; S\left(\mathbb{R}^{n}\right)\right)$ при $\rho \geqslant \rho_{*}$ справедливо неравенство

$$
\rho \int_{-T}^{T}\|v\|^{2} e^{2 \rho \phi(t)} d t \leqslant 3 \int_{-T}^{T}\left\|\left(D_{t}-\varkappa\left(x, t, D_{x}\right)\right) v\right\|^{2} e^{2 \rho \phi(t)} d t
$$

әде

$$
\|v\|=\left(\int_{\mathbb{R}^{n}}|v(x, t)|^{2} d x\right)^{1 / 2}
$$

ДокАЗАтЕльство. Для функций $w \in C_{0}^{\infty}\left((-T, T) ; S\left(\mathbb{R}^{n}\right)\right)$ справедливо доказываемое интегрированием по частям тождество

$$
\begin{aligned}
\int_{-T}^{T} & \left\|\left(D_{t}+i \rho \phi_{t}^{\prime}-\varkappa\left(x, t, D_{x}\right)\right) w\right\|^{2} d t \\
= & \int_{-T}^{T}\left\|\left(D_{t}-B_{1}\right) w\right\|^{2} d t+\int_{-T}^{T}\left\|\left(i \rho \phi_{t}^{\prime}-i B_{2}\right) w\right\|^{2} d t \\
& \quad+\rho \int_{-T}^{T}\|w\|^{2} d t-i \int_{-T}^{T}\left(\left[D_{t}-B_{1}, B_{2}\right] w, w\right) d t
\end{aligned}
$$

где $B_{1}=\left(\varkappa+\varkappa^{*}\right) / 2, B_{2}=\left(\varkappa-\varkappa^{*}\right) /(2 i),(\cdot, \cdot)$ - скалярное произведение в $L_{2}\left(\mathbb{R}^{n}\right)$ и $[\cdot, \cdot]$ - коммутатор операторов.

Если символ оператора $\varkappa\left(x, t, D_{x}\right)$ удовлетворяет условию $\left(\mathrm{i}^{\prime}\right)$, то в силу теоремы 2.1 символы операторов $B_{2}$ и $\left[D_{t}-B_{1}, B_{2}\right]$ принадлежат классу $S_{w}^{0}$ и, следовательно, операторы ограничены из $L_{2}\left(\mathbb{R}^{n}\right)$ в $L_{2}\left(\mathbb{R}^{n}\right)$. В этом случае из тождества (2.11) вытекает неравенство

$$
\int_{-T}^{T}\left\|\left(D_{t}+i \rho \phi_{t}^{\prime}-\varkappa\left(x, t, D_{x}\right)\right) w\right\|^{2} d t \geqslant \rho\left(1-\frac{C}{\rho}\right) \int_{-T}^{T}\|w\|^{2} d t
$$

где $C$ - некоторая константа, зависящая от операторов $B_{1}$ и $B_{2}$. Выбирая $\rho_{*}=$ $3 C / 2$, а $\delta_{2}>0$ - произвольньм числом, меньшим $T_{1}$, и подставляя в последнее неравенство $w=v e^{\rho \phi}$, получаем доказьваемое утверждение в случае $\left(\mathrm{i}^{\prime}\right)$.

В случае (ii') для оператора $B_{2}$ с помошью хорошо известного в теории псевдодифференциальных операторов способа можно построить почти обратный квазиполиоднородный псевдодифференциальный оператор $B_{3}$ с символом класса $S_{w}^{-\mu}$. Разность $B_{3} \circ B_{2}-\mathrm{Id}$, где Id - тождественный оператор, является псевдодифференциальным оператором с символом класса $S_{w}^{0}$. Отсюда следует, что сушествует константа $C$, с которой для всех $w \in C_{0}^{\infty}\left((-T, T) ; S\left(\mathbb{R}^{n}\right)\right)$ равномерно по $t \in(-T, T)$, $T<T_{1}$, выполняется оценка

$$
\|w\|_{\mu} \leqslant C\left(\left\|B_{2} w\right\|+\|w\|\right)
$$


где $\|\cdot\|_{\mu}$ - норма в пространстве $H^{\mu}$. Так как $\left|\phi_{t}^{\prime}\right| \leqslant 3 T$ при $t \in(-T, T)$, отсюда следует, что

$$
\|w\|_{\mu} \leqslant C\left(\left\|\left(i \rho \phi_{t}^{\prime}-i B_{2}\right) w\right\|+(3 T \rho+1)\|w\|\right) .
$$

Коммутатор $\left[D_{t}-B_{1}, B_{2}\right]$ является псевдодифференциальным оператором с символом класса $S_{w}^{\mu}$. Из последнего неравенства и теоремы 2.1 нетрудно вывести оценку

$$
\left|\left(\left[D_{t}-B_{1}, B_{2}\right] w, w\right)\right| \leqslant\left\|\left(i \rho \phi_{t}^{\prime}-i B_{2}\right) w\right\|^{2}+C(T \rho+1)\|w\|^{2},
$$

где $C$ - некоторая новая положительная константа. Теперь из тождества (2.11) получаем

$$
\int_{-T}^{T}\left\|\left(D_{t}+i \rho \phi_{t}^{\prime}-\varkappa\left(x, t, D_{x}\right)\right) w\right\|^{2} d t \geqslant \rho\left(1-C T-\frac{C}{\rho}\right) \int_{-T}^{T}\|w\|^{2} d t .
$$

Выбирая $\delta_{2}=C / 3$ и $\rho_{*}=3 C$ и полагая в последнем неравенстве $w=v e^{\rho \phi}$, получаем доказываемое утверждение в случае $\left(\mathrm{ii}^{\prime}\right)$.

Основное неравенство. Если каждый из операторов $\varkappa^{j}=\varkappa^{j}\left(x, t, D_{x}\right)$, $j=1,2, \ldots, k$, удовлетворяет одному из условий $\left(\mathrm{i}^{\prime}\right)$ или $\left(\mathrm{ii}^{\prime}\right)$, то из леммы 2.2 следует, что существуют числа $\delta_{2} \in\left(0, T_{1}\right)$ и $\rho_{*}$ такие, что для всех $T \in\left(0, \delta_{2}\right]$ и векторнозначных функций $\mathbf{v} \in C_{0}^{\infty}\left((-T, T) ; S\left(\mathbb{R}^{n}\right)\right) \otimes \mathbb{C}^{k}$ при $\rho \geqslant \rho_{*}$ справедливо неравенство

$$
\rho \int_{-T}^{T}\|\mathbf{v}\|^{2} e^{2 \rho \phi(t)} d t \leqslant 3 \int_{-T}^{T}\left\|\left(D_{t}-\operatorname{diag}\left(\varkappa^{1}, \ldots, \varkappa^{k}\right)\right) \mathbf{v}\right\|^{2} e^{2 \rho \phi(t)} d t .
$$

Для каждого числа $s \in \mathbb{R}$ определим на $C_{0}^{\infty}\left((-T, T) \times \mathbb{R}^{n}\right)$ норму

$$
\|u\|_{s, T}=\left(\sum_{j=0}^{k-1} \int_{-T}^{T}\left\|\Lambda(D)^{m-\mu(j+1)} D_{t}^{j} u\right\|_{s}^{2} e^{2 \rho \phi(t)} d t\right)^{1 / 2} .
$$

ЛЕМма 2.3. Для любого числа $\varepsilon>0$ существует окрестность $U$ начала координат такая, что для всех чисел $\rho>0$ и функиий $u \in C_{0}^{\infty}((-T, T) \times U)$ справедливо неравенство

$$
\|u\|_{-\mu, T}^{2} \leqslant\left(\varepsilon+\frac{1}{T^{2} \rho^{2}}\right)\|u\|_{0, T}^{2} .
$$

ДоКАЗАТЕЛЬСТво. По построению функции $\phi(t)$ при $t \in(-T, T)$ имеет место оценка

$$
e^{2 \rho \phi(t)} \leqslant-\frac{1}{2 T \rho} \frac{\partial}{\partial t} e^{2 \rho \phi(t)}
$$

из которой интегрированием по частям при $j=0,1, \ldots, k-2$ легко выводится неравенство

$$
\begin{aligned}
\int_{-T}^{T}\left\|\Lambda(D)^{m-\mu(j+2)} D_{t}^{j} u\right\|^{2} e^{2 \rho \phi(t)} d t & \\
& \leqslant \frac{1}{T^{2} \rho^{2}} \int_{-T}^{T}\left\|\Lambda(D)^{m-\mu(j+2)} D_{t}^{j+1} u\right\|^{2} e^{2 \rho \phi(t)} d t
\end{aligned}
$$


$u \in C_{0}^{\infty}\left((-T, T) \times \mathbb{R}^{n}\right)$. В силу анизотропного аналога теоремы 3.12 из работы Ю.В. Егорова [6; гл. 1] для любого $\varepsilon>0$ найдется окрестность $U$ начала координат такая, что для всех функций $u \in C_{0}^{\infty}((-T, T) \times U)$ справедливо неравенство

$$
\int_{-T}^{T}\left\|\Lambda(D)^{-\mu} D_{t}^{k-1} u\right\|^{2} e^{2 \rho \phi(t)} d t \leqslant \varepsilon \int_{-T}^{T}\left\|D_{t}^{k-1} u\right\|^{2} e^{2 \rho \phi(t)} d t .
$$

У тверждение леммы является прямьм следствием определения нормы $\|\cdot\|_{-\mu, T}$ и приведенных оценок.

ЛЕмма 2.4. Пусть дифференциальный оператор (2.1) удовлетворяет условию $\left(1^{\prime}\right)$ и для каждой точки $\left(0,0, \xi^{0}, 0\right), \xi^{0} \neq 0$, существует окрестность $W$, в которой решения $\tau_{j}(x, t, \xi, h), j=1,2, \ldots, k$, уравнения

$$
\mathscr{H}_{P}(x, t, \xi, \tau, h)=0
$$

удовлетворяют одному из условий $\left(\mathrm{I}^{\prime}\right)$ или $\left(\mathrm{II}^{\prime}\right)$. Тогда существуют окрестность $U \subset \mathbb{R}^{n}$ начала координат и число $\delta_{2}>0$ такие, что для каждого $T \in\left(0, \delta_{2}\right]$ для всех функиий $\left.u \in C_{0}^{\infty}((-T, T) \times U)\right)$ с некоторой константой $C>0$ при $\rho \geqslant \rho_{*}(T)$ выполняется оценка

$$
\rho \sum_{j=0}^{k-1} \int_{-T}^{T}\left\|\Lambda(D)^{m-\mu(j+1)} D_{t}^{j} u\right\|^{2} e^{2 \rho \phi(t)} d t \leqslant C \int_{-T}^{T}\|P u\|^{2} e^{2 \rho \phi(t)} d t
$$

әде $\|\cdot\|-$ норма в $L_{2}\left(\mathbb{R}^{n}\right), \rho_{*}(T)$ - число, зависящее от $T$.

ДокАзАтЕльство. Из проведенных вьше рассуждений следует, что для любого $\xi^{0} \in\left\{\xi:|\xi|_{\varrho}=1\right\}$ сушествуют окрестность $U_{\xi^{0}} \subset \mathbb{R}^{n}$ начала координат, открытая квазиконическая окрестность $\Gamma_{\xi^{0}} \subset \mathbb{R}^{n}$ точки $\xi^{0}$, положительные числа $\delta_{2, \xi^{0}}, \rho_{*, \xi^{0}}$ и семейства матричных псевдодифференциальных операторов $E_{\xi^{0}}$ и $\operatorname{diag}\left(\varkappa_{\xi^{0}}^{1}, \ldots, \varkappa_{\xi^{0}}^{k}\right)$ такие, что справедливы соотношения $(2.9),(2.10)$ и (2.12). Символ семейства операторов $E_{\xi^{0}}$ является бесконечно дифференцируемой функцией от $t \in\left(-\delta_{2, \xi^{0}}, \delta_{2, \xi^{0}}\right)$ со значениями в $S_{w}^{0} \otimes\left(\mathbb{C}^{k} \otimes \mathbb{C}^{k}\right)$. Уменњшая при необходимости $\delta_{2, \xi^{0}}$ и используя $(2.9)$, с помощью обьчной для теории псевдодифференциальных операторов техники можно построить такое семейство псевдодифференциальных операторов $R_{\xi^{0}}$ с символом, являющимся бесконечно дифференцируемой функцией от $t \in\left(-\delta_{2, \xi^{0}}, \delta_{2, \xi^{0}}\right)$ со значениями в $S_{w}^{0} \otimes\left(\mathbb{C}^{k} \otimes \mathbb{C}^{k}\right)$, для которого символы семейств операторов $\left(E_{\xi^{0}} \circ R_{\xi^{0}}-I\right)$ и $\left(R_{\xi^{0}} \circ E_{\xi^{0}}-I\right)$ будут бесконечно диффференцируемыми функциями от $t$ со значениями в $S_{w}^{-\mu} \otimes\left(\mathbb{C}^{k} \otimes \mathbb{C}^{k}\right)$.

Из покрытия множества $\left\{\xi:|\xi|_{\varrho}=1\right\}$ открытыми множествами вида $\Gamma_{\xi^{0}}$ выберем конечное подпокрытие $\Gamma_{l}=\Gamma_{\xi}$, где $l=1, \ldots, N$. Положим $\Gamma_{0}=\left\{\xi:|\xi|_{\varrho}<1\right\}$. Множества $\left\{\Gamma_{l}\right\}_{l=0}^{N}$ образуют открытое покрытие пространства $\mathbb{R}^{n}$. Пусть $\left\{\psi_{l}(\xi)\right\}_{l=0}^{N}-$ семейство бесконечно дифференцируемых функций, подчиненных покрытию $\left\{\Gamma_{l}\right\}_{l=0}^{N}$, таких, что

$$
\sum_{l=0}^{N} \psi_{l}^{2}(\xi)=1, \quad \xi \in \mathbb{R}^{n}
$$


причем функции $\psi_{l}(\xi)$ при $l=1, \ldots, N$ положительно квазиоднородны нулевой степени при больших $\xi$. Положим $V=\bigcap_{l>0} U_{\xi^{l}}, \delta_{2}=\min _{l>0}\left\{\delta_{2, \xi^{l}}\right\}$.

Из свойств псевдодифференциальных операторов для любого $l \geqslant 1$ первое слагаемое $S_{1, l}=R_{l} \circ\left(E_{l} \circ \operatorname{diag}\left(\varkappa_{l}^{1}, \ldots, \varkappa_{l}^{k}\right)+A \circ E_{l}\right) \circ R_{l}$ в правой части операторного тождества

$$
\begin{aligned}
& R_{l} \circ\left(D_{t}+A\right)-\left(D_{t}-\operatorname{diag}\left(\varkappa_{l}^{1}, \ldots, \varkappa_{l}^{k}\right)\right) \circ R_{l} \\
= & S_{1, l}+\left[R_{l}, D_{t}\right]-R_{l} \circ A \circ\left(E_{l} \circ R_{l}-I\right)-\left(E_{l} \circ R_{l}-I\right) \circ \operatorname{diag}\left(\varkappa_{l}^{1}, \ldots, \varkappa_{l}^{k}\right) \circ R_{l}
\end{aligned}
$$

является семейством псевдодифференциальных операторов, символ которого есть бесконечно дифференцируемая функция от $t \in\left(-\delta_{2}, \delta_{2}\right)$ со значениями в $\left(S_{w}^{\mu} \cap S_{w}^{0}\left(V \times \Gamma_{l}\right)\right) \otimes\left(\mathbb{C}^{k} \otimes \mathbb{C}^{k}\right)$. Оставшшеся слагаемые образуют семейство матричных псевдодифференциальных операторов, символ которого есть бесконечно дифференцируемая функция от $t \in\left(-\delta_{2}, \delta_{2}\right)$ со значениями в $S_{w}^{0} \otimes\left(\mathbb{C}^{k} \otimes \mathbb{C}^{k}\right)$.

При $l \geqslant 1$ для всех функций $\mathbf{v} \in C_{0}^{\infty}\left((-T, T) ; S\left(\mathbb{R}^{n}\right)\right) \otimes \mathbb{C}^{k}$ при $\rho \geqslant \rho_{*, l}(T)$ с некоторой константой $C$ справедливо неравенство

$$
\begin{aligned}
\rho \int_{-T}^{T} & \left\|\psi_{l}(D) \mathbf{v}\right\|^{2} e^{2 \rho \phi(t)} d t \\
\leqslant & C\left(\int_{-T}^{T}\left\|\psi_{l}(D)\left(D_{t}+A\right) \mathbf{v}\right\|^{2} e^{2 \rho \phi(t)} d t\right. \\
& \left.\quad+\int_{-T}^{T}\|\mathbf{v}\|^{2} e^{2 \rho \phi(t)} d t+\int_{-T}^{T}\left\|S_{1, l} \psi_{l}(D) \mathbf{v}\right\|^{2} e^{2 \rho \phi(t)} d t\right) .
\end{aligned}
$$

В самом деле, из свойств семейства операторов $R_{l}$ следует, что с некоторой константой $C$ имеет место оценка

$$
\begin{aligned}
& \rho \int_{-T}^{T}\left\|\psi_{l}(D) \mathbf{v}\right\|^{2} e^{2 \rho \phi(t)} d t \\
& \leqslant \rho C\left(\int_{-T}^{T}\left\|R_{l} \psi_{l}(D) \mathbf{v}\right\|^{2} e^{2 \rho \phi(t)} d t+\int_{-T}^{T}\left\|\psi_{l}(D) \mathbf{v}\right\|_{-\mu}^{2} e^{2 \rho \phi(t)} d t\right) .
\end{aligned}
$$

Используя лемму 2.2 , операторное тождество и теорему 2.1 , правую часть последнего неравенства можно оценить сверху произведением некоторой константы на выражение

$$
\begin{aligned}
\int_{-T}^{T} \| \psi_{l}(D)\left(D_{t}+A\right) & \mathbf{v}\left\|^{2} e^{2 \rho \phi(t)} d t+\int_{-T}^{T}\right\| \mathbf{v} \|^{2} e^{2 \rho \phi(t)} d t \\
& +\int_{-T}^{T}\left\|S_{1, l} \psi_{l}(D) \mathbf{v}\right\|^{2} e^{2 \rho \phi(t)} d t+\rho \int_{-T}^{T}\|\mathbf{v}\|_{-\mu}^{2} e^{2 \rho \phi(t)} d t
\end{aligned}
$$

Теперь, оценивая последнее слагаемое полученного выражения с помощью леммы 2.3, можно завершить доказательство неравенства (2.14).

Заметим, что при $l=0$ оценка (2.14) также имеет место, причем без последнего слагаемого в правой части неравенства. Это вытекает из компактности носителя 
функции $\psi_{0}(\xi)$ и справедливости оценки (2.12) в тривиальном случае, когда все операторы $\varkappa^{j}$ нулевые.

Пусть открытая окрестность начала координат $U$ имеет компактное замыкание, содержашееся в $V$. Для функции $u \in C_{0}^{\infty}((-T, T) \times U)$ положим

$$
v^{j}=\Lambda^{m-\mu j}(D) D_{t}^{j-1} u, \quad j=1, \ldots, k .
$$

Тогда из свойств семейств операторов $S_{1, l}$ следует, что существует такая константа $C$, с которой для всех $u \in C_{0}^{\infty}((-T, T) \times U)$ и $l \geqslant 1$ справедлива оценка

$$
\left\|S_{1, l} \psi_{l}(D) \mathbf{v}\right\|^{2} \leqslant C \sum_{j=0}^{k-1}\left\|\Lambda(D)^{m-\mu(j+1)} D_{t}^{j} u\right\|^{2}
$$

Используя (2.14) и очевидное равенство

$$
\sum_{j=0}^{k-1}\left\|\Lambda(D)^{m-\mu(j+1)} D_{t}^{j} u\right\|^{2}=\sum_{l=0}^{N}\left\|\psi_{l}(D) \mathbf{v}\right\|^{2}
$$

выбирая константу $C$ и число $\rho_{*}(T)$ достаточно большими, нетрудно завершить доказательство леммы.

Через $H_{w, \text { comp }}^{s}(\Omega)$ обозначим подпространство в $H_{w}^{s}(\Omega)$, состоящее из элементов, имеющих компактные носители, содержащиеся в открытом множестве $\Omega \subseteq \mathbb{R}^{n}$.

ТЕОРема 2.5. Пусть дифференциальный оператор (2.1) удовлетворяет условию $\left(1^{\prime}\right)$ и для каждой точки $\left(0,0, \xi^{0}, 0\right), \xi^{0} \neq 0$, существует окрестность $W$, в которой решения $\tau_{j}(x, t, \xi, h), j=1,2, \ldots, k$, уравнения

$$
\mathscr{H}_{P}(x, t, \xi, \tau, h)=0
$$

удовлетворяют одному из условий $\left(\mathrm{I}^{\prime}\right)$ или $\left(\mathrm{II}^{\prime}\right)$. Тогда существуют окрестность $U \subset \mathbb{R}^{n}$ начала координат и число $\delta_{2}>0$ такие, что для каждого $T \in\left(0, \delta_{2}\right]$ для всех функиий $u \in H_{w, \operatorname{comp}}^{m-\mu}((-T, T) \times U)$ с Pu $\in L_{2}((-T, T) \times U)$ при $\rho \geqslant \rho_{*}(T)$ выполняется оценка (2.2) с некоторой константой $C>0$, где $\rho_{*}(T)$ - некоторое число, зависящее от $T$.

ДокАЗАТЕЛЬСТво. С помощью леммы Фридрихса [5] доказательство сводится к тому случаю, когда $u \in C_{0}^{\infty}((-T, T) \times U)$. Тогда утверждение теоремы вытекает из леммы 2.4, свойств преобразования Фурье и элементарной оценки

$$
\left|\xi^{\alpha}\right| \leqslant C_{\alpha} \Lambda(\xi)^{s}, \quad \xi \in \mathbb{R}^{n}
$$

справедливой при $\langle\varrho, \alpha\rangle \leqslant s$, где $C_{\alpha}-$ константа, зависяшая от $s$ и $\alpha$. 


\section{§3. Доказательство теорем о продолжении решения через гиперповерхность}

Теорема 2.5, которой мы предполагаем воспользоваться при доказательстве теоремы 1.1, применима к финитным функциям. Технически удобно от функции $u$ из теоремы 1.1 перейти к финитной умножением на срезающую функцию специального вида $\chi(\varphi(x))$. Прием дает нужный результат, если пересечение гиперповерхности $S$ с носителем $u$ состоит не более чем из одной точки $x^{0}$. В связи с этим исходная гиперповерхность $S$ будет заменена гиперповерхностью $S_{1}=\left\{x \in U_{1}\right.$ : $\left.\varphi_{1}(x)=0\right\}$, близкой к $S$ в точке $x^{0} \in S \cap S_{1}$ в том смысле, что $\varphi_{1, x}\left(x^{0}\right)=\varphi_{x}\left(x^{0}\right)$. Если в точке $x^{0}$ для оператора $P$ вида (1.1) относительно $S$ выполнялись условия нехарактеристичности поверхности, простоты корней (1), а также условие (2), описываюшее допустимые типы корней, то все эти условия сохраняют силу для $S_{1}$ в точке $x^{0}$. В самом деле, непосредственно из равенства $\varphi_{1, x}\left(x^{0}\right)=\varphi_{x}\left(x^{0}\right)$ вытекают нехарактеристичность $S_{1}$ в точке $x^{0}$ и выполнение условия (1) для $S_{1}$ в точке $x^{0}$. Сохранение типов корней легко доказать с помощью теоремы о неявной функции.

Для того чтобы воспользоваться теоремой 2.5, нужно оператор $P$ вида (1.1) заменой координат привести к специальному виду (2.1). Не ограничивая обшности, можно считать, что точка $x^{0}$ есть начало координат. Поскольку поверхность $S$ является нехарактеристической в точке $x^{0}$, то для некоторого номера $j$ такого, что $\varrho_{j}=\mu$, производная $\frac{\partial \varphi}{\partial x_{j}}$ в точке $x^{0}$ отлична от нуля. Пусть для определенности $\frac{\partial \varphi}{\partial x_{n+1}}(0) \neq 0$ и $\varrho_{n+1}=\mu$. Сделаем замену координат $y_{j}=x_{j}$ при $j=1, \ldots, n$, $t=\varphi(x)$. Переменной $t$ поставим в соответствие вес $\mu$, за остальными переменными сохраним прежние веса. В результате преобразования оператор $P$ вида (1.1) с точностью до отличного от нуля в окрестности начала координат множителя $\left.p_{m}\left(x, \varphi_{x^{\prime}}(x), 0\right)\right|_{x=x(y, t)}$ перейдет в оператор $P_{1}$ вида $(2.1)$. Оператор $P_{1}$ определен в окрестности начала координат. Для того чтобы получить оператор, определенный в полосе вида $\Pi=\mathbb{R}^{n} \times\left(-T_{0}, T_{0}\right)$, достаточно продолжить коэффициенты $a_{\alpha, j}(t, x)$ при $j=0, \ldots, k-1$ из локального представления (2.1) на полосу. Последнее легко осуществить, умножая коэффициенты на срезающие функции.

Пусть $\eta, \tau$ - двойственные переменные по отношению к переменным $y, t$. Между пучками символов $\mathscr{H}_{P}(x, \xi, h)$ и $\mathscr{H}_{P_{1}}(y, t, \eta, \tau, h)$ операторов $P$ и $P_{1}$ в окрестности точки $y=0, t=0$ имеется легко проверяемая связь

$$
\begin{aligned}
\mathscr{H}_{P_{1}}(y, t, \eta, \tau, h) p_{m}\left(x(y, t), \varphi_{x^{\prime}}(x(y, t)), 0\right) \\
\quad=\mathscr{H}_{P}(x(y, t), \xi(y, t, \eta, \tau, h), h)+O\left(h^{\mu}\right),
\end{aligned}
$$

где $\xi_{j}(y, t, \eta, \tau, h)=\eta_{j}+h^{\varrho_{j}-\mu} \varphi_{x_{j}}(x(y, t)) \tau, j=1, \ldots, n$, и $\xi_{n+1}(y, t, \eta, \tau, h)=$ $\varphi_{x_{n+1}}(x(y, t)) \tau$. Остаток $O\left(h^{\mu}\right)$ является произведением степени $h^{\mu}$ на многочлен относительно $\eta, \tau, h$ с бесконечно дифференцируемыми по $y$ и $t$ коэффициентами. В силу (3.1) если для оператора $P$ в начале координат выполняется условие (1), то для оператора $P_{1}$ в начале координат выполняется условие $\left(1^{\prime}\right)$. Далее, из (3.1) следует, что в некоторой квазиконической окрестности $W$ точки $y=0, t=0$, 
$\eta^{0} \neq 0, h=0$ при всех $\tau \in \mathbb{C}$ справедливо сравнение

$$
\prod_{j=1}^{k}\left(\tau-\tau_{j}(y, t, \eta, h)\right)=\prod_{j=1}^{k}\left(\tau-z_{j}(x(y, t), \xi(\eta), h)\right)+O\left(h^{\mu}\right)
$$

где $\xi_{j}(\eta)=\eta_{j}$ при $j=1, \ldots, n$ и $\xi_{n+1}=0$. Отсюда вытекает, что если каждый из корней уравнения (1.2) удовлетворяет одному из условий (I) или (II), то каждый корень $\tau_{j}(y, t, \eta, h)$ удовлетворяет одному из условий $\left(\mathrm{I}^{\prime}\right)$ или $\left(\mathrm{II}^{\prime}\right)$. В самом деле, подставим на место $\tau$ корень $z_{l}(x(y, t), \xi(\eta), h)$, где $l$ - фиксированный номер. Тогда

$$
\prod_{j=1}^{k}\left(z_{l}(x(y, t), \xi(\eta), h)-\tau_{j}(y, t, \eta, h)\right)=O\left(h^{\mu}\right)
$$

Поскольку корни $\tau_{j}\left(0,0, \eta^{0}, 0\right)$ попарно различны, то для каждого $l=1,2, \ldots, k$ найдется один номер $j(l)$ такой, что $\tau_{j(l)}\left(0,0, \eta^{0}, 0\right)=z_{l}\left(x^{0}, \xi\left(\eta^{0}\right), 0\right)$. Не ограничивая общности, можно считать, что $j(l)=l$. Очевидно, если для $z_{l}$ вьполняется условие (II), то для $\tau_{l}$ выполняется $\left(\mathrm{II}^{\prime}\right)$. Далее, дифференцируя сравнение по $h$ и затем полагая $h=0$, последовательно получаем, что $\frac{\partial^{r} \tau_{l}}{\partial h^{r}}(y, t, \eta, 0)=$ $\frac{\partial^{r} z_{l}}{\partial h^{r}}(x(y, t), \xi(\eta), 0)$ при $r=0,1, \ldots, \mu-1$. Поэтому если для $z_{l}$ выполняется условие $(\mathrm{I})$, то для $\tau_{l}$ выполняются равенства, составляющие условие $\left(\mathrm{I}^{\prime}\right)$.

ДоКАЗАТЕЛЬСТво ТЕОРЕМЫ 1.1. В обозначениях, предшествующих доказательству, заменим функцию $\varphi(x)$, определяющую гиперповерхность $S$, проходящую через точку $x^{0}=0$, на функцию $\varphi(x)+\sum_{j=1}^{n} x_{j}^{2}$. При этом, как уже отмечалось, в точке $x^{0}$ свойства (1) и (2) сохраняют силу. После замены получим, что $u=0$ в точках $x \in U$, в которых $\varphi(x)<\sum_{j=1}^{n} x_{j}^{2}$. Затем сделаем указанную выше локальную замену координат, приводящую оператор $P$ к виду (2.1). При этом определенньй в некоторой окрестности начала координат локальный обратный образ $\widetilde{u}(y, t)$ обобщенной функции $u(x)$ удовлетворяет уравнению $P_{1} \widetilde{u}=0$. Кроме того, носитель supp $\widetilde{u}$ содержится в пересечении этой окрестности с множеством $t \geqslant|y|^{2}$. Уменьшая в случае необходимости число $T_{0}$ и продолжая $\widetilde{u}(y, t)$ нулем на дополнение окрестности в полосе П, можно считать, что обобщенная функция $\widetilde{u}(y, t)$ принадлежит $H_{w}^{m-\mu}(\Pi), P_{1} \widetilde{u}=0$ в П и $\operatorname{supp} \widetilde{u} \subset\left\{(y, t) \in \Pi: t \geqslant|y|^{2}\right\}$.

Выберем теперь положительное число $T$ так, чтобы множество $\{(y, t) \in \Pi$ : $\left.t \geqslant|y|^{2}, t \leqslant T\right\}$ содержалось в произведении $U \times\left(-\delta_{2}, \delta_{2}\right)$, где $U$ и $\delta_{2}-$ окрестность начала координат и число из теоремы 2.5. Для того чтобы применить теорему 2.5, умножим функцию $\widetilde{u}(y, t)$ на подходящую срезающую функцию. Пусть функция $\chi(t) \in C^{\infty}(R)$ удовлетворяет равенству $\chi(t)=1$ при $t \leqslant 2 T_{1}$ и равенству $\chi(t)=0$ при $t \geqslant T_{2}$, где $0<2 T_{1}<T_{2}<T$.

Применим теорему 2.5 к функции $\chi \widetilde{u} \in H_{w, \operatorname{comp}}^{m-\mu}(U \times(-T, T))$. Заметим, что $P_{1}(\chi \widetilde{u})=\left[P_{1}, \chi(t)\right] \widetilde{u}$. Коммутатор $\left[P_{1}, \chi(t)\right]$ оператора $P_{1}$ и оператора умножения на функцию $\chi(t)$ является дифференциальным оператором, взвешенный порядок которого не превосходит $m-\mu$, а коэффициенты равны нулю вне полосы 
$2 T_{1}<t<T_{2}$. Функция $\phi(t)$ монотонно убывает на интервале $(-T, T)$. Поэтому из теоремы 2.5 следует, что для функции $\widetilde{u}$ с некоторой константой $C$ при $\rho \geqslant \rho_{*}(T)$ имеет место оценка

$$
\rho \sum_{\langle\varrho, \alpha\rangle \leqslant m-\mu} \int_{0}^{T_{1}}\left\|D_{t, y}^{\alpha} \widetilde{u}\right\|^{2} d t \leqslant C e^{2 \rho\left(\phi\left(2 T_{1}\right)-\phi\left(T_{1}\right)\right)} \sum_{\langle\varrho, \alpha\rangle \leqslant m-\mu} \int_{2 T_{1}}^{T_{2}}\left\|D_{t, y}^{\alpha} \widetilde{u}\right\|^{2} d t .
$$

Поскольку $\phi\left(2 T_{1}\right)<\phi\left(T_{1}\right)$, то для того чтобы это неравенство выполнялось при $\rho \rightarrow+\infty$, необходимо, чтобы

$$
\sum_{\langle\varrho, \alpha\rangle \leqslant m-\mu} \int_{0}^{T_{1}}\left\|D_{t, y}^{\alpha} \widetilde{u}\right\|^{2} d t=0
$$

Отсюда вытекает, что $u(x)=0$ в некоторой окрестности начала координат. Теорема доказана.

ДоКАЗАТЕЛЬСТво ТЕОРЕмы 1.2. Функция $u$, продолженная нулем на $U \backslash \Omega$, является решением однородного уравнения в $U$. Отсюда и из теоремы 1.1 следует доказываемое утверждение.

ДОКАЗАТЕЛЬСТВо ТЕОРЕМЫ 1.3. Из рассуждений, приведенных при доказательстве теоремы 1.1 , следует, что для произведения обратного образа $\widetilde{u}(y, t)$ функции $u(x)=u_{1}(x)-u_{2}(x)$ на срезающую функцию $\chi(t)$ справедлива теорема 2.5. При замене координат нелинейный дифференциальный оператор $F\left(x, D^{\beta} u\right)$ преобразуется в нелинейный дифференциальный оператор $\widetilde{F}\left(x, D^{\beta} \widetilde{u}\right)$ с сохранением взвешенного порядка и условия $(3)$ из $\S 1$ относительно отображения $\widetilde{F}$. Из теоремы 2.5 следует, что для функции $\widetilde{u}$ с некоторой константой $C$ при $\rho \geqslant \rho_{*}(T)$ справедлива оценка

$$
\begin{aligned}
& \rho \sum_{\langle\varrho, \alpha\rangle \leqslant m-\mu} \int_{0}^{2 T_{1}}\left\|D_{t, y}^{\alpha} \widetilde{u}\right\|^{2} e^{2 \rho \phi(t)} d t \\
& \quad \leqslant C \sum_{\langle\varrho, \alpha\rangle \leqslant m-\mu}\left(\int_{2 T_{1}}^{T_{2}}\left\|D_{t, y}^{\alpha} \widetilde{u}\right\|^{2} e^{2 \rho \phi(t)} d t+\int_{0}^{2 T_{1}}\left\|D_{t, y}^{\alpha} \widetilde{u}\right\|^{2} e^{2 \rho \phi(t)} d t\right),
\end{aligned}
$$

где $0<2 T_{1}<T_{2}<T$. Увеличивая число $\rho_{*}(T)$, из последнего неравенства получаем, что при всех $\rho>\rho_{*}(T)$ с некоторой новой константой $C$ имеет место оценка

$$
\rho \sum_{\langle\varrho, \alpha\rangle \leqslant m-\mu} \int_{0}^{T_{1}}\left\|D_{t, y}^{\alpha} \widetilde{u}\right\|^{2} d t \leqslant C e^{2 \rho\left(\phi\left(2 T_{1}\right)-\phi\left(T_{1}\right)\right)} \sum_{\langle\varrho, \alpha\rangle \leqslant m-\mu} \int_{2 T_{1}}^{T_{2}}\left\|D_{t, y}^{\alpha} \widetilde{u}\right\|^{2} d t .
$$

Так же как и при доказательстве теоремы 1.1 , устремляя $\rho \mathrm{k}+\infty$, отсюда получаем, что $u(x)=0$ в некоторой окрестности начала координат. Теорема доказана. 
ДокАЗАТЕЛЬСТво ТЕОРЕмЫ 1.4. Поскольку $d \varphi \neq 0$ на $U$, поверхность $S=$ $\{x \in U: \varphi(x)=0\}$ делит $U$ на две части: $U_{+}=\{x \in U: \varphi(x)>0\}$ и $U_{-}=\{x \in U: \varphi(x)<0\}$. Покажем, что $u_{1}=u_{2}$ в пересечении $U_{+}$с некоторой окрестностью точки $x^{0}$. Определим в $U$ функцию $u$, положив $u=u_{1}-u_{2}$ в $U_{+}$ и $u=0$ в $U_{-}$. Тогда функция $u \in C_{w}^{m-\mu}(\Omega)$ обращается в нуль в $U_{-}$и как обобщенная функция удовлетворяет уравнению

$$
P(x, D) u=\theta(\varphi(x))\left(F\left(x, D^{\beta} u_{1}\right)-F\left(x, D^{\beta} u_{2}\right)\right),
$$

где $\theta(s)$ - функция Хевисайда. В оставшейся части доказательство можно провести по схеме доказательства теоремы 1.3. Аналогично можно показать, что $u_{1}=u_{2}$ в пересечении $U_{-}$с некоторой окрестностью точки $x^{0}$. Теорема доказана.

Прежде чем переходить к доказательству теоремы 1.5, докажем две леммы относительно свойств введенного в $\S 1$ множества $\Xi$. Доказательство леммы 3.1 следует плану, намеченному Л. Хёрмандером в [1] для изотропного случая.

ЛЕмМа 3.1. Множсество $\Xi$ является квазиконическим замкнутым в $\Omega \times$ $\left(\mathbb{R}^{n_{1}} \backslash\{0\}\right)$ подмножеством.

ДокАЗАТЕЛЬСТво. Из квазиоднородности по $\xi$ символа $p_{m}(x, \xi)$ легко выводится, что $\Xi-$ квазиконус.

Множество точек $\left(x, \eta^{\prime}\right), \eta^{\prime} \neq 0$, в которых $p_{m}\left(x, \eta^{\prime}, 0\right)=0$, содержится в $\Xi$. Если $p_{m}\left(x, \eta^{\prime}, 0\right) \neq 0$, то $\left(x, \eta^{\prime}\right) \in \Xi$ тогда и только тогда, когда сушествует ненулевой вектор $\xi$ такой, что многочлен $p_{m}\left(x, \xi^{\prime}+z \eta^{\prime}, \xi^{\prime \prime}\right)$ имеет корень $z^{0}$ кратности не ниже 2 , причем $\xi+z\left(\eta^{\prime}, 0\right) \neq 0$. Используя квазиоднородность символа $p_{m}(x, \xi)$, отсюда можно вьвести, что при указанных предположениях относительно $\left(x, \eta^{\prime}\right)$ включение $\left(x, \eta^{\prime}\right) \in \Xi$ имеет место тогда и только тогда, когда существует такой вектор $\xi$, что $|\xi|_{\varrho}=1, \sum_{j \in J^{\prime}} \xi_{j} \eta_{j}=0$ и многочлен $p_{m}\left(x, \xi^{\prime}+z \eta^{\prime}, \xi^{\prime \prime}\right)$ имеет корень $z^{0}$ кратности не ниже 2 . Теперь, используя компактность множества $\left\{\xi:|\xi|_{\varrho}=1, \sum_{j \in J^{\prime}} \xi_{j} \eta_{j}=0\right\}$, можно показать, что для любой точки $\left(x, \eta^{\prime}\right) \notin \Xi$ найдется окрестность $V \subset \Omega \times\left(\mathbb{R}^{n_{1}} \backslash\{0\}\right)$, имеющая пустое пересечение с $\Xi$, что завершает доказательство леммы.

Обозначим через $\Xi_{1}$ множество точек $\left(x, \eta^{\prime}\right) \in \Omega \times\left(\mathbb{R}^{n_{1}} \backslash\{0\}\right)$, для которых существует вектор $\xi \in \mathbb{R}^{n}$, не коллинеарный вектору $\left(\eta^{\prime}, 0\right)$, такой, что полином $p_{m}\left(x, \xi^{\prime}+z \eta^{\prime}, \xi^{\prime \prime}\right)$ по $z \in \mathbb{C}$ имеет по крайней мере один корень $z^{0}$ кратности выше единицы.

Лемма 3.2. Если $p_{m}\left(x, \eta^{\prime}, 0\right) \neq 0$, mо $\left(x, \eta^{\prime}\right) \in \Xi$ тогда и только тогда, когда $\xi \in \Xi_{1}$.

ДокАЗАтЕльство. Если $\left(x, \eta^{\prime}\right) \in \Xi$ и $\left(x, \eta^{\prime}\right) \notin \Xi_{1}$, то сушествует такой вектор $\xi$, что уравнение $p_{m}\left(x, \xi^{\prime}+z \eta^{\prime}, \xi^{\prime \prime}\right)=0$ относительно $z$ имеет корень $z^{0}$ кратности не ниже $2, \xi+z^{0}\left(\eta^{\prime}, 0\right) \neq 0$ и $\xi=\vartheta\left(\eta^{\prime}, 0\right), \vartheta \in \mathbb{R}$. Из однородности $p_{m}\left(x, \xi^{\prime}, 0\right)$ вытекает, что $p_{m}\left(x, \xi^{\prime}+z \eta^{\prime}, \xi^{\prime \prime}\right)=(\vartheta+z)^{m / \mu} p_{m}\left(x, \eta^{\prime}, 0\right)$. Следовательно, $z^{0}=-\vartheta$, что противоречит условию $\xi+z^{0}\left(\eta^{\prime}, 0\right) \neq 0$.

Если $\left(x, \eta^{\prime}\right) \in \Xi_{1}$ и $\left(x, \eta^{\prime}\right) \notin \Xi$, то сушествует такой вектор $\xi$, что уравнение $p_{m}\left(x, \xi^{\prime}+z \eta^{\prime}, \xi^{\prime \prime}\right)=0$ относительно $z$ имеет корень $z^{0}$ кратности не ниже 2 , вектор $\xi$ неколлинеарен вектору $\left(\eta^{\prime}, 0\right)$ и $\xi+z^{0}\left(\eta^{\prime}, 0\right)=0$. Последнее равенство противоречит неколлинеарности векторов $\xi$ и $\left(\eta^{\prime}, 0\right)$. Лемма доказана. 
ДОКАЗАТЕЛЬСТВО ТЕОРЕМЫ 1.5. Предположим противное: $x^{0} \in \operatorname{supp} u$. По условию теоремы $\operatorname{supp} u \cap U \subseteq\{x \in U: \varphi(x) \geqslant 0\}$. В силу предложения 8.5.8 из монографии Л. Хёрмандера [1] найдутся последовательности открытых множеств $U_{j}$, функций $f_{j}(x) \in C^{\infty}\left(U_{j}\right)$ и точек $x^{j}, j=1,2, \ldots$, такие, что $x^{j} \in \operatorname{supp} u$, $\left(x^{j}, f_{j, x}\left(x^{j}\right)\right) \rightarrow\left(x^{0},-\varphi_{x}\left(x^{0}\right)\right)$ при $j \rightarrow+\infty$ и $\operatorname{supp} u \cap U_{j} \subseteq\left\{x \in U_{j}: f_{j}(x) \leqslant\right.$ $\left.f_{j}\left(x^{j}\right)\right\}$. Можно считать, что $f_{j}\left(x^{j}\right)=0$. Положим $\varphi_{j}(x)=-f_{j}(x)$. В силу леммы 3.1 множество $\Xi$ замкнуто в $\Omega \times\left(\mathbb{R}^{n_{1}} \backslash\{0\}\right)$, и по условию теоремы точка $\left(x^{0}, \varphi_{x^{\prime}}\left(x^{0}\right)\right)$ не принадлежит $\Xi$. Поэтому $\left(x^{j}, \varphi_{j, x^{\prime}}\left(x^{j}\right)\right) \notin \Xi$ при достаточно больших $j$. Далее, из сходимости $\varphi_{j, x}\left(x^{j}\right) \rightarrow \varphi_{x}\left(x^{0}\right)$ следует, что при больших $j$ каждая гиперповерхность $S_{j}=\left\{x \in U_{j}: \varphi_{j}(x)=0\right\}$ является нехарактеристической для оператора $P(x, D)$ в точке $x^{j}$. Кроме того, в силу леммы 3.2 при больших $j$ в точках $x^{j}$ для каждой поверхности $S_{j}$ выполняются условие простоты корней (1) и условия (I), (II) на типы корней уравнения

$$
\mathscr{H}\left(x, \xi+h^{\varrho-\mu} z \varphi_{j, x}(x), h\right)=0 .
$$

Рассуждениями, аналогичными приведенньм при доказательстве теоремы 1.3 , можно показать, что $x^{j} \notin \operatorname{supp} u$ при больших $j$. Полученное противоречие завершает доказательство теоремы.

\section{§4. Об однозначном продолжении по части переменных ростков решений}

Если все решения линейного уравнения $P u=0$ в $\Omega$ являются функциями, аналитическими по переменным $x^{\prime}$, то сужение любого решения на плоскость вида $x^{\prime \prime}=x^{\prime \prime 0}$, где $x^{\prime \prime}$ - дополнение $x^{\prime}$ до полной совокупности координат и $x^{\prime \prime 0}$ - заданные числа, имеет однозначное продолжение из произвольной окрестности $U$ точки $\left(x^{\prime 0}, x^{\prime \prime 0}\right)$ на связную компоненту пересечения указанной плоскости с $\Omega$, содержащую точку $\left(x^{\prime 0}, x^{\prime \prime 0}\right)$. Для однородных параболических уравнений с аналитическими по пространственным переменным коэффициентами решения являются аналитическими по пространственным переменньм функциями (см., например, [11]). Для уравнений с неаналитическими коэффициентами однозначность продолжения ростков решений доказана для параболических уравнений при некоторых дополнительных предположениях относительно оператора, порождающего уравнение [12].

Следует отметить, что одного условия параболичности уравнения для справедливости утверждения об однозначном продолжении ростка решения по пространственным переменным недостаточно. В самом деле, Плись [2; теорема 2] построил линейный однородный эллиптический оператор $L$ в $\mathbb{R}^{3}$ четвертого порядка, для которого уравнение $L u=0$ имеет нетривиальное решение $u_{0}(x)$ с компактным носителем. Коэффициенты оператора вешественны и бесконечно дифференцируемы. Функция $u(t, x)=u_{0}(x)$ является решением параболического уравнения

$$
\frac{\partial u}{\partial t}-L u=0, \quad(t, x) \in \mathbb{R}^{4} .
$$

Поскольку $\operatorname{supp} u=\mathbb{R}_{t} \times \operatorname{supp}_{x} u_{0}$, то для данного параболического уравнения утверждение об однозначном продолжении по пространственньм переменным (вдоль любой характеристики $t=t_{0}$ ) не может быть справедливым. 
Полученные выше теоремы о локальной единственности решений позволяют доказать однозначную продолжимость ростков решений по части переменных для ряда линейных и квазилинейных уравнений с неаналитическими коэффициентами.

Будем предполагать, что определенный в открытом множестве $\Omega$ оператор $P$ вида (1.1) удовлетворяет следующим условиям:

(Р.1) для любой точки $x^{0} \in \Omega$ всякая проходящая через нее гиперповерхность $S=\{x \in U: \varphi(x)=0\}$ такая, что $\varphi_{x^{\prime}}\left(x^{0}\right) \neq 0$, является нехарактеристической в $x^{0}$ для $P$;

(Р.2) для любой точки $x^{0} \in \Omega$, вектора $\eta^{\prime 0} \in \mathbb{R}^{n_{1}}$ такого, что $p_{m}\left(x^{0}, \eta^{\prime 0}, 0\right) \neq 0$, и вектора $\xi^{0} \in \mathbb{R}^{n+1}$, не коллинеарного $\left(\eta^{\prime 0}, 0\right)$, многочлен $p_{m}\left(x^{0}, \xi^{\prime 0}+\right.$ $\left.z \eta^{\prime 0}, \xi^{\prime \prime 0}\right)$ по переменной $z$ не имеет кратных корней.

Из условия (Р.2) и теоремы о неявной функции следует, что для каждой точки $\left(x^{0}, \eta^{\prime 0}, \xi^{0}\right) \in \Omega \times \mathbb{R}^{n_{1}} \times \mathbb{R}^{n+1}$, удовлетворяющей требованиям (Р.2), найдется окрестность точки $\left(x^{0}, \eta^{\prime 0}, \xi^{0}, 0\right)$, в которой уравнение

$$
\mathscr{H}_{P}\left(x, \xi^{\prime}+z \eta^{\prime}, \xi^{\prime \prime}, h\right)=0
$$

относительно $z$ имеет $k=m / \mu$ простых корней $z_{j}\left(x, \eta^{\prime}, \xi, h\right)$. Будем предполагать, что

(Р.3) каждый из корней уравнения удовлетворяет одному из условий:

(P.I) $\operatorname{Im} z_{j}\left(x, \eta^{\prime}, \xi, h\right) \equiv 0$ в некоторой окрестности точки $\left(x^{0}, \eta^{\prime 0}, \xi^{0}, 0\right)$;

(P.II) $\operatorname{Im} z_{j}\left(x^{0}, \eta^{\prime 0}, \xi^{0}, 0\right) \neq 0$.

Если оператор $P$ удовлетворяет условиям (Р.1)-(Р.3), то в любой точке $x^{0} \in \Omega$ поверхность $S=\{x \in U: \varphi(x)=0\}$ такая, что $\varphi_{x^{\prime}}\left(x^{0}\right) \neq 0$, является нехарактеристической для $P$ и вьполняются условия (1) и (2).

Оператор $P$ назьвается $\xi^{\prime}$-квазиэллиптическим в точке $x$, если из равенств $p_{m}(x, \xi)=0$ и $\xi^{\prime \prime}=0$ следует, что $\xi^{\prime}=0$. Оператор $P$ называется $\xi^{\prime}$-квазиэллиптическим в $\Omega$, если он является $\xi^{\prime}$-квазиэллиптическим в любой точке $\Omega$.

ПРЕДЛОЖЕНИЕ 4.1. Оператор $P$ удовлетворяет условию (Р.1) тогда и только тогда, когда он является $\xi^{\prime}$-квазиэллиптическим в $\Omega$.

Равносильность условия (Р.1) и $\xi^{\prime}$-квазиэллиптичности очевидна.

ПРЕДЛОЖЕНИЕ 4.2. Оператор $P$ удовлетворяет условию (Р.2) тогда и только тогда, когда он удовлетворяет аналогичному условию, в котором требование неколлинеарности векторов $\xi^{0} u\left(\eta^{\prime 0}, 0\right)$ заменено на условие ортогональности $\sum_{j \in J^{\prime}} \xi_{j}^{\prime 0} \eta_{j}^{\prime 0}=0$ и условие $\left|\xi^{0}\right|_{\varrho}=1$.

ДокаЗАтельство. Необходимость очевидна. Докажем достаточность. Предположим, что условие (Р.2) не выполняется. В этом случае для некоторых неколлинеарных векторов $\xi^{0}$ и $\left(\eta^{\prime 0}, 0\right)$ система

$$
\left\{\begin{array}{l}
p_{m}\left(x^{0}, \xi^{\prime 0}+z \eta^{\prime 0}, \xi^{\prime \prime 0}\right)=0 \\
\sum_{j \in J^{\prime}} p_{m, \xi_{j}^{\prime}}\left(x^{0}, \xi^{\prime 0}+z \eta^{\prime 0}, \xi^{\prime \prime 0}\right) \eta_{j}^{\prime 0}=0
\end{array}\right.
$$

имеет решение $z^{0} \in \mathbb{C}$. Тогда из квазиоднородности $p_{m}$ следует, что для любых чисел $\lambda>0$ и $\delta \in \mathbb{R}$ аналогичная система с $\xi^{0}$, замененным на $\widetilde{\xi}^{0}=\lambda \circ\left(\xi^{0}+\delta\left(\eta^{\prime 0}, 0\right)\right)$, 
и $\eta^{\prime 0}$, замененным на $\widetilde{\eta}^{\prime 0}=\lambda^{\mu} \eta^{\prime 0}$, имеет решение $z^{0}-\delta$. Выберем $\lambda$ и $\delta$ так, чтобы $\sum_{j \in J^{\prime}} \widetilde{\xi}_{j}^{\prime 0} \widetilde{\eta}_{j}^{\prime 0}=0$ и $\left|\widetilde{\xi}^{0}\right|_{\varrho}=1$. Это возможно, так как $\xi^{0}$ и $\left(\eta^{\prime 0}, 0\right)$ неколлинеарны. Сушествование $\widetilde{\xi}^{0}$ и $\widetilde{\eta}^{\prime 0}$ противоречит условию, сформулированному в доказываемом предложении.

ПРЕДЛОЖЕНИЕ 4.3. Если оператор Р удовлетворяет условию (Р.2) и взвешенные символь $p_{j}(x, \xi)$ вещественны при $j=m, \ldots, m-\mu+1$, то оператор $P$ удовлетворяет условию (Р.3).

ДокАЗАТЕЛЬСтво. Поскольку при $j=m, \ldots, m-\mu+1$ символы $p_{j}(x, \xi)$ вешественны, то по определению пучка $\mathscr{H}_{P}$ коэффициенты уравнения (4.1) будут вешественными при $\left(x, \eta^{\prime}, \xi, h\right) \in \Omega \times \mathbb{R}^{n_{1}} \times \mathbb{R}^{n+1} \times \mathbb{R}$. Поэтому если $z^{0}$ есть вешественный корень уравнения при $x=x^{0}, \xi=\xi^{0}, \eta^{\prime}=\eta^{\prime 0}$ и $h=0$, то в силу (Р.2) и теоремы о неявной функции (вещественный случай) найдутся некоторая окрестность точки $\left(x^{0}, \eta^{\prime 0}, \xi^{0}, 0\right)$ и определенная в ней вешественнозначная бесконечно дифференцируемая функция $z\left(x, \eta^{\prime}, \xi, h\right)$, которая удовлетворяет уравнению в точках окрестности и $z\left(x^{0}, \eta^{\prime 0}, \xi^{0}, 0\right)=z^{0}$. Следовательно, в этом случае выполняется (P.I). Если $\operatorname{Im} z^{0} \neq 0$, то, очевидно, имеет место (Р.II).

Для каждой точки $x^{0}$ открытого множества $\Omega$ через $F_{\Omega}\left(x^{0}\right)$ обозначим связную компоненту слоя $\left\{x \in \Omega: x^{\prime \prime}=x^{\prime \prime 0}\right\}$, содержащую $x^{0}$.

Теорема 4.4. Пусть $P(x, D)$ удовлетворяет условиям (P.1)-(Р.3). Тогда если функиия $u \in \mathscr{D}^{\prime}(\Omega)$ является решением уравнения $P(x, D) u=0$ в $\Omega$, $u \in H_{w}^{m-\mu}(\Omega) u x^{0} \notin \operatorname{supp} u$, mo $F_{\Omega}\left(x^{0}\right) \cap \operatorname{supp} u=\varnothing$.

ДокАЗАтельство. Допустим, что множество $F_{\Omega}\left(x^{0}\right)$ содержит точку $y^{0} \in$ $\operatorname{supp} u$. Пусть $\gamma:[0,1] \rightarrow F_{\Omega}\left(x^{0}\right)-$ непрерывный путь, соединяющий точки $x^{0}$ и $y^{0}$. Так как $x^{0} \notin \operatorname{supp} u$, то найдется наибольшее число $s^{*} \in(0,1]$ такое, что $\gamma(t) \notin \operatorname{supp} u$ при всех $t \in\left[0, s^{*}\right)$. Очевидно, $\gamma\left(s^{*}\right) \in \operatorname{supp} u$ и в любой окрестности этой точки найдутся точки $\gamma(t) \notin \operatorname{supp} u$. Отсюда следует, что множество $F_{\Omega}\left(x^{0}\right)$ включает в себя отрезок прямой $I$, содержащий две внутренние точки, одна из которых принадлежит $\operatorname{supp} u$, а другая нет. Сужая в случае необходимости отрезок, можно добиться того, что середина отрезка не будет принадлежать $\operatorname{supp} u$. Чтобы не усложнять обозначения, будем считать, что середина отрезка совпадает с началом координат и отрезок $I$ содержится в координатной оси $x_{1}$. В частности, из сделанного предположения следует, что $\varrho_{1}=\mu$.

Вследствие замкнутости носителя $\operatorname{supp} u$ найдется положительное число $r$ такое, что множество

$$
\left\{x:\left|x_{1}\right|<r, \sum_{j=2}^{n+1} x_{j}^{2}<r\right\}
$$

содержится в $\Omega$ и имеет пустое пересечение с supp $u$. Выберем положительные числа $r_{1}<r$ и $r_{2}$ так, что при каждом $a \in(0,1]$ эллипсоид

$$
\mathscr{E}_{a}=\left\{x: \varphi(x) \equiv \frac{x_{1}^{2}}{r_{2}^{2}}+\sum_{j=2}^{n+1} \frac{x_{j}^{2}}{r_{1}^{2}}=a\right\}
$$


содержится в $\Omega$ и $I \cap \mathscr{E}_{1}=\varnothing$. Так как $\varrho_{1}=\mu$, то в силу (Р.1) при $a \in(0,1]$ любая точка $x^{0} \in \mathscr{E}_{a}$, у которой первая координата отлична от нуля, является нехарактеристической для оператора $P$. По условиям теоремы в каждой такой точке с $\eta^{\prime 0}=\varphi_{x^{\prime}}\left(x^{0}\right)$ для оператора $P$ выполняются условия (Р.2) и (Р.3). По построению найдется наименњшее число $a^{*} \in(0,1]$ такое, что эллипсоид $\mathscr{E}_{a^{*}}$ содержит точку $x^{*} \in \operatorname{supp} u$, причем $x_{1}^{*} \neq 0$. Поскольку $\mathscr{E}_{a} \cap \operatorname{supp} u=\varnothing$ при $a<a^{*}$, то в силу теоремы $1.1 u=0$ в некоторой окрестности точки $x^{*}$. Полученное противоречие доказывает теорему.

В силу теоремы 4.4 росток в точке $x^{0}$ достаточно гладкого решения уравнения, удовлетворяющего условиям теоремы, однозначно определяет решение в некоторой окрестности множества $F_{\Omega}\left(x^{0}\right)$.

Для точки $x^{0}$, принадлежащей гранище множества $\Omega$, через $F_{\partial \Omega}\left(x^{0}\right)$ обозначим связную компоненту слоя $\left\{x \in \Omega: x^{\prime \prime}=x^{\prime \prime 0}\right\}$, замыканию которой принадлежит $x^{0}$. Следует отметить, что множество $F_{\partial \Omega}\left(x^{0}\right)$ может оказаться пустьм.

Tеорема 4.5. Пусть оператор $P(x, D)$ определен в открытом множестве $\Omega \cup U$, где $U$ - некоторая открытая окрестность точки $x^{0} \in \partial \Omega$, и удовлетворяет на этом множсестве условиям (Р.1)-(Р.3). Предположим, что через точку $x^{0}$ можно провести гиперповерхность $S=\{x \in U: \varphi(x)=0\}$ такую, что $\varphi(x)>\varphi\left(x^{0}\right)$ при $x \in U \cap \Omega$ u $\varphi_{x^{\prime}}\left(x^{0}\right) \neq 0$. Тогда если функиия $u \in \mathscr{D}^{\prime}(\Omega)$ является решением уравнения $P(x, D) u=0$ в $\Omega$ и $u \in \dot{H}_{w}^{m}\left(x^{0}\right) \cap H_{w}^{m-\mu}(\Omega), m o F_{\partial \Omega}\left(x^{0}\right) \cap \operatorname{supp} u=\varnothing$.

Доказательство следует из теорем 1.2 и 4.4 .

ПримеР 1.В качестве примера рассмотрим линейный дифференциальный оператор с бесконечно дифференцируемыми коэффициентами

$$
P=\frac{\partial}{\partial t}-\sum_{j, l=1}^{n} a_{j, l}(t, x) \frac{\partial^{2}}{\partial x_{j} \partial x_{l}}+P_{1}\left(t, x, D_{x}\right),
$$

где $A(t, x)=\left(a_{j, l}(t, x)\right)$ - симметрическая $(n \times n)$-матрица, $P_{1}\left(t, x, D_{x}\right)$ - оператор первого порядка. Поставим в соответствие дифференцированию по $t$ вес 2 , а дифференцированию по $x$ - вес 1 . Взвешенный главный символ оператора имеет вид

$$
p_{2}=i \tau+\langle A(t, x) \xi, \xi\rangle .
$$

Если для любой точки $(t, x) \in \Omega$ уравнение $\operatorname{Re}\langle A(t, x) \xi, \xi\rangle=0$ имеет единственное решение $\xi=0$, то в силу предложения 4.1 для $P$ выполняется условие (Р.1). Покажем, что в этом случае для $P$ вьполняются условия (Р.2) и (Р.3). Опуская у матрицы $A$ аргументы $t$ и $x$, разложим ее на вешественную и мнимую части: $A=A_{1}+i A_{2}$. Не ограничивая общности, можно считать, что квадратичная форма, порожденная матрицей $A_{1}$, положительно определена. Пусть $\eta \neq 0$. Условие (Р.2) достаточно проверить для векторов $(\tau, \xi)$, удовлетворяющих равенству $\left\langle A_{1} \eta, \xi\right\rangle=0$. Для того чтобы квадратный трехчлен $p_{2}(t, x, \tau, \xi+z \eta)$ имел кратный корень, необходимо и достаточно, чтобы его дискриминант равнялся нулю. Приравнивая к нулю вешественную и мнимую части дискриминанта, получаем

$$
\left\{\begin{array}{l}
-\left\langle A_{2} \eta, \xi\right\rangle^{2}-\left\langle A_{1} \xi, \xi\right\rangle\left\langle A_{1} \eta, \eta\right\rangle+\left(\tau+\left\langle A_{2} \xi, \xi\right\rangle\right)\left\langle A_{2} \eta, \eta\right\rangle=0 \\
\left(\tau+\left\langle A_{2} \xi, \xi\right\rangle\right)\left\langle A_{1} \eta, \eta\right\rangle+\left\langle A_{1} \xi, \xi\right\rangle\left\langle A_{2} \eta, \eta\right\rangle=0
\end{array}\right.
$$


Исключая $\tau$, получаем равенство, из которого вследствие положительной определенности формы $\left\langle A_{1} \eta, \eta\right\rangle$ вытекает, что $\left\langle A_{1} \xi, \xi\right\rangle=0$ и, следовательно, $\xi=0$. Отсюда и из второго равенства системы получаем, что $\tau=0$. Оператор $P$ удовлетворяет условию (Р.2). Если $z^{0}$ - вешественный корень трехчлена $p_{2}(t, x, \tau, \xi+z \eta)$, то $\left\langle A_{1}\left(\xi+z^{0} \eta\right), \xi+z^{0} \eta\right\rangle=0$. Отсюда следует, что $\xi+z^{0} \eta=0$ и $\tau=0$, т.е. векторы $(\tau, \xi)$ и $(0, \eta)$ коллинеарны. Таким образом, в данном случае для корней уравнения (4.1) выполняется условие (P.II). Из теоремы 4.4 следует, что два решения $u_{1}, u_{2} \in \mathscr{D}^{\prime}(\Omega)$ уравнения $P u=f$ в $\Omega$, совпадающие в некоторой окрестности точки $\left(x^{0}, t^{0}\right) \in \Omega$ и такие, что $\left(u_{1}-u_{2}\right) \in H_{w}^{1}(\Omega)$, будут равны в некоторой окрестности связной компоненты слоя $F_{\Omega}\left(\left(x^{0}, t^{0}\right)\right)=\left\{(x, t) \in \Omega: t=t^{0}\right\}$. В частности, если $f=0$ и $u_{2}=0$, то $u_{1}=0$ в некоторой окрестности связной компоненты слоя $F_{\Omega}\left(\left(x^{0}, t^{0}\right)\right)$. Если в точке $\left(x^{0}, t^{0}\right) \in \partial \Omega$ выполняются условия теоремы 4.5 и $u_{1}, u_{2}$ удовлетворяют условиям теоремы, то $u_{1}=u_{2}$ в некоторой окрестности множества $F_{\partial \Omega}\left(\left(x^{0}, t^{0}\right)\right)$. В том случае, когда $f=0$ и $u_{2}=0$, получаем, что решение $u_{1} \in \dot{H}_{w}^{2}\left(\left(x^{0}, t^{0}\right)\right) \cap H_{w}^{1}(\Omega)$ уравнения $P u=0$ в $\Omega$ равно нулю в некоторой окрестности множества $F_{\partial \Omega}\left(\left(x^{0}, t^{0}\right)\right)$, если последнее множество непусто.

ПримеР 2. В качестве второго примера рассмотрим на произведении $\Omega=$ $(-\varepsilon, T) \times \Omega_{1}$, где $\Omega_{1}$ - открытое множество в $\mathbb{R}^{n}, \varepsilon, T$ - положительные числа, линейный дифференциальный оператор с бесконечно дифференцируемыми коэффициентами

$$
P=D_{t}^{3}-\sum_{j, l=1}^{n} a_{j, l}(t, x) \frac{\partial^{2}}{\partial x_{j} \partial x_{l}}+P_{1}\left(t, x, D_{t}, D_{x}\right),
$$

$P_{1}\left(t, x, D_{t}, D_{x}\right)$ - дифференциальньй оператор второго порядка по совокупности переменных $t, x$ и первого по $x$. Поставим в соответствие дифференцированию по $t$ вес 2 , а дифференцированию по $x$ - вес 3 . Взвешенньй главный символ оператора $P$ имеет вид

$$
p_{6}=\tau^{3}+\langle A(t, x) \xi, \xi\rangle=\tau^{3}+\sum_{j, l=1}^{n} a_{j, l}(t, x) \xi_{j} \xi_{l} .
$$

Оператор $P$ удовлетворяет (Р.1). Если для любой точки $(t, x) \in \Omega$ уравнение $\langle A(t, x) \xi, \xi\rangle=0$ имеет единственное решение $\xi=0$, то для оператора $P$ выполняется условие (Р.2). Для выполнения (Р.3) достаточно, чтобы символы $p_{6}$ и $p_{5}$ были вещественны или чтобы в каждой точке $(t, x) \in \Omega$ квадратичная форма $\operatorname{Im}\langle A(t, x) \xi, \xi\rangle$ была отлична от нуля при $\xi \neq 0$. Предположим, что оператор $P$ удовлетворяет условиям (Р.1)-(Р.3).

Пусть функция $u(t, x)$ является классическим решением задачи Коши

$$
\begin{aligned}
P u & =0, \quad(t, x) \in(0, T) \times \Omega_{1} \\
\frac{\partial^{l} u}{d t^{k}}(0, x) & =\psi_{l}(x), \quad x \in \Omega_{1}, \quad l=0,1,2
\end{aligned}
$$

Пусть $x^{0} \notin \bigcup_{l=0}^{2} \operatorname{supp} \psi_{l}(x)$. Выберем открытую окрестность $U$ точки $\left(0, x^{0}\right)$ в $\Omega$ так, чтобы пересечение $U$ с плоскостью $t=0$ не содержало точек, принадлежаших носителям функций $\operatorname{supp} \psi_{l}(x), l=0,1,2$. Продолжая $u(t, x)$ нулем на $U_{-}=$ $\{(t, x) \in U: t<0\}$, получаем определенное в $U$ и обращающееся в нуль в $U_{-}$ 
решение уравнения $P u=0$. Из теоремы 1.1 следует, что $u=0$ в некоторой окрестности точки $\left(0, x^{0}\right)$. В силу теоремы $4.4 u=0$ в некоторой окрестности множества $(0, T) \times x^{0}$, т.е.

$$
\operatorname{supp} u \subseteq(0, T) \times \bigcup_{l=0}^{2} \operatorname{supp} \psi_{l}
$$

Таким образом, если в окрестности точки $x^{0} \in \Omega_{1}$ начальные данные Коши обрашаются в нуль, то решение $u(t, x)$ указанной задачи Коши равно нулю в некоторой окрестности точки $\left(0, x^{0}\right)$. Подобный эффект имеет место для обькновенных дифференциальных уравнений, зависящих от параметра.

ТЕОрема 4.6. Пусть $P(x, D)$ удовлетворяет условиям (P.1)-(Р.3) и непрерывное отображсение $F: \Omega \times \mathbb{C}^{N_{m-\mu}} \rightarrow \mathbb{C}$ удовлетворяет условию (3) (см. 1 ). Тогда если $u_{1}, u_{2} \in C_{w}^{m-\mu}(\Omega)$ являются решениями уравнения

$$
P(x, D) u=F\left(x, D^{\beta} u\right), \quad x \in \Omega,
$$

и $u_{1}=u_{2}$ в некоторой окрестности точки $x^{0}$, то

$$
F_{\Omega}\left(x^{0}\right) \cap \operatorname{supp}\left(u_{1}-u_{2}\right)=\varnothing .
$$

ДокАЗАтЕльство. Положим $u=u_{1}-u_{2}$. Теперь теорема доказывается по той же схеме, что и теорема 4.4. Отличие состоит в том, что на заключительном этапе следует воспользоваться теоремой 1.3 вместо теоремы 1.1 .

СлЕДСТВИЕ 4.7. Пусть $P(x, D)$ удовлетворяет условиям (Р.1)-(P.3), непрерьвное отображсение $F: \Omega \times \mathbb{C}^{N_{m-\mu}} \rightarrow \mathbb{C}$ удовлетворяет условию (3) и $F(x, 0)=0$. Тогда если $и \in C_{w}^{m-\mu}(\Omega)$ является решением уравнения

$$
P(x, D) u=F\left(x, D^{\beta} u\right), \quad x \in \Omega,
$$

$u$ и 0 в некоторой окрестности точки $x^{0}$, то

$$
F_{\Omega}\left(x^{0}\right) \cap \operatorname{supp} u=\varnothing \text {. }
$$

ДокАЗАТЕльство. В данном случае функция $u_{2}=0$ является решением уравнения. Применяя теорему 4.6, получаем доказываемое утверждение.

Для справедливости следствия достаточно, чтобы условие (3) выполнялось при $\zeta^{2}=0$.

ТЕОРЕМА 4.8. Предположим, что выполнены условия теоремы 4.6 и поверхность $S$ в точке $x^{0}$ не является характеристической для оператора $P(x, D)$. Тогда если функиии $u_{1}, u_{2} \in C_{w}^{m}(\Omega)$ являются решениями уравнения (4.2) и $\left.D^{\alpha}\left(u_{1}-u_{2}\right)\right|_{S}=0$ nрu $\langle\varrho, \alpha\rangle \leqslant m-\mu$, то

$$
F_{\Omega}\left(x^{0}\right) \cap \operatorname{supp}\left(u_{1}-u_{2}\right)=\varnothing .
$$


ДокаЗАТЕЛЬство. Положим $u=u_{1}-u_{2}$. Теперь теорема доказывается так же, как теорема 4.4. Вместо теоремы 1.1 на заключительном этапе следует воспользоваться теоремой 1.4 .

ПримеР 3. В математических моделях при описании физико-химических и биологических процессов часто используется уравнение

$$
\frac{\partial u}{\partial t}-\sum_{j, l=1}^{n} a_{j, l}(t, x) \frac{\partial^{2} u}{\partial x_{j} \partial x_{l}}+F\left(t, x, u, \frac{\partial u}{\partial x}\right)=0
$$

Первоначально уравнение при $F=F(u)$ было выведено А.Н. Колмогоровым, И.Г. Петровским и И.С. Пискуновым в работе [13] из гипотетических предположений для описания временнь́х изменений пространственно распределенной плотности вешества при наличии диффузии и роста количества вещества. Позже это уравнение получено Я.Б. Зельдовичем [14] в связи с изучением распространения пламени. В дальнейшем волновые свойства решений уравнений активно исследовались на протяжении последних пятидесяти лет различными авторами.

Теорема 4.6 позволяет утверждать, что при не очень ограничительных условиях на нелинейную часть, содержащихся в (3), для достаточно гладких решений имеет место однозначное продолжение ростков решений по пространственным переменньм. Точнее, пусть коэффициенты $a_{j, l}(t, x)$ являются бесконечно диффференцируемыми функциями в открытом множестве $\Omega$. Введем взвешивание так же, как в примере 1. Пусть для любой точки $(t, x) \in \Omega$ уравнение $\operatorname{Re}\langle A(t, x) \xi, \xi\rangle=0$ имеет единственное решение $\xi=0$. Тогда для оператора, определяющего линейную часть уравнения, вьполняются условия (Р.1)-(Р.3). Пусть непрерывное отображение $F: \Omega \times \mathbb{C}^{n+1} \rightarrow \mathbb{C}$ удовлетворяет условию (3). Тогда если функции $u_{1}, u_{2} \in C(\Omega)$ имеют непрерывные по $x$ первые производные, как обобщенные функции являются решениями уравнения (4.3) и $u_{1}=u_{2}$ в некоторой окрестности точки $\left(t^{0}, x^{0}\right)$, то

$$
F_{\Omega}\left(\left(t^{0}, x^{0}\right)\right) \cap \operatorname{supp}\left(u_{1}-u_{2}\right)=\varnothing .
$$

Предположим, что $F(t, x, 0,0)=0$. Тогда если решение $u_{1}$ обращается в нуль в некоторой окрестности точки $\left(t^{0}, x^{0}\right)$, то $u_{1}=0$ в некоторой окрестности связной компоненты слоя $\left\{(t, x) \in \Omega: t=t^{0}\right\}$, содержащей точку $\left(t^{0}, x^{0}\right)$. Дополнительно предположим, что множество $\Omega$ расслаивается на связные подмножества $\Omega_{t^{0}}=$ $\left\{(t, x) \in \Omega: t=t^{0}\right\}$. Тогда если в каждом слое найдется точка $\left(t^{0}, x\left(t^{0}\right)\right)$ такая, что $u_{1}=0$ в некоторой ее окрестности, то $u_{1}=0$ в $\Omega$.

ПРИмеР 4. Предположим, что в уравнении

$$
u_{t}+u_{x x x}+F\left(x, t, u, u_{x}, u_{x x}\right)=0
$$

обобщаюшем уравнение Кортевега-де $\Phi$ риза $(\mathrm{K} д \Phi)$, нелинейный член $F$ удовлетворяет условию (3). Введем взвешивание производных, присвоив однократному дифференцированию по $t$ вес 3 , по $x$ - вес 1 . Линейная часть уравнения (4.4) определяется оператором, удовлетворяющим условиям (Р.1)-(Р.3). Поэтому из теоремы 4.6 получаем, что два непрерывных решения $u_{1}$ и $u_{2}$, имеющих непрерывные 
производные по $x$ первого и второго порядков и совпадающих в некоторой окрестности точки $\left(x^{0}, t^{0}\right)$, совпадают в окрестности связной компоненты $F_{\Omega}\left(\left(x^{0}, t^{0}\right)\right)$ слоя $\Omega_{t^{0}}=\left\{(x, t) \in \Omega: t=t^{0}\right\}$, содержащей точку $\left(x^{0}, t^{0}\right)$. Предположим дополнительно, что $F(x, t, 0,0,0)=0$. Тогда если решение $u$, удовлетворяюшее указанньм выше условиям гладкости, обращается в нуль в некоторой окрестности точки $\left(x^{0}, t^{0}\right)$, то оно равно нулю в окрестности множества $F_{\Omega}\left(\left(x^{0}, t^{0}\right)\right)$. При $\Omega=\mathbb{R} \times(0, T)$ отсюда получаем, что равное нулю в окрестности точки $\left(x^{0}, t^{0}\right)$ решение равно нулю в окрестности слоя $\mathbb{R} \times\left\{t^{0}\right\}$. Таким образом, если каждьй слой $\mathbb{R} \times\left\{t^{0}\right\}$ содержит точку, в окрестности которой решение равно нулю, то решение тождественно равно нулю. В предположении большей гладкости решений уравнения (4.4) можно пользоваться теоремой 4.8. Пусть функции $u_{1}$ и $u_{2}$ имеют непрерывные производные первого порядка по $t$ и до третьего порядка включительно по $x$ и являются решениями уравнения (4.4). Тогда если для нехарактеристической поверхности $S$ при $k=0,1,2$ выполняются равенства $\left.\frac{\partial^{k}}{\partial x^{k}}\left(u_{1}-u_{2}\right)\right|_{S}=0$, то $u_{1}=u_{2}$ в окрестности каждого множества $F_{\Omega}\left(\left(x^{0}, t^{0}\right)\right)$, где $\left(x^{0}, t^{0}\right) \in S$.

ПРимеР 5. В уравнении типа Кадомцева-Петвиашвили [15]

$$
u_{x_{1} x_{1} x_{1} x_{1}}+3 u_{x_{2} x_{2}}+u_{x_{1} x_{3}}+F\left(x, u, u_{x}, u_{x_{1} x_{1}}, u_{x_{1} x_{2}}, u_{x_{1} x_{1} x_{1}}\right)=0
$$

введем взвешивание производных, положив $\varrho_{1}=1, \varrho_{2}=2$ и $\varrho_{3}=3$. Взвешенный главный символ оператора $P$ линейной части уравнения имеет вид $p_{4}=\xi_{1}^{4}-$ $3 \xi_{2}^{2}-\xi_{1} \xi_{3}$. Очевидно, что для оператора $P$ выполняется условие (Р.1). Проверка выполнения условия (Р.2) проводится прямой выкладкой. Из предложения 4.3 следует, что для $P$ выполняется условие (Р.3). Предположим, что непрерывное отображение $F: \Omega \times \mathbb{C}^{7} \rightarrow \mathbb{C}$, порождающее нелинейный член уравнения (4.5), удовлетворяет условию (3) из $§ 1$. Тогда из теоремы 4.6 получаем, что непрерывные решения $u_{1}$ и $u_{2}$, имеюшие непрерывные производные, указанные в числе аргументов нелинейной части уравнения, и совпадающие в некоторой окрестности точки $x^{0}$, совпадают в окрестности связной компоненты $F_{\Omega}\left(x^{0}\right)$ слоя $\Omega_{x_{2}^{0}, x_{3}^{0}}=\left\{x \in \Omega: x_{2}=x_{2}^{0}, x_{3}=x_{3}^{0}\right\}$, содержашей точку $x^{0}$. Предположим дополнительно, что $F(x, 0,0,0,0,0)=0$. Тогда если решение $u$, удовлетворяюшее указанньм выше условиям гладкости, обращается в нуль в некоторой окрестности точки $x^{0}$, то оно равно нулю в окрестности множества $F_{\Omega}\left(x^{0}\right)$.

В заключение заметим, что теорема 4.6 допускает следуюшую интерпретацию. Пусть выполняются условия теоремы. Тогда множество $C_{w}^{m-\mu}(\Omega)$-решений уравнения (4.2) образует класс квазианалитических функций по переменным минимального веса.

\section{Список литературы}

1. Хёрмандер Л. Анализ линейных диффференциальных операторов с частньми производными. Т. 1-4. М.: Мир, 1987.

2. Plis A. Linear elliptic differential equations without any solution in a sphere // Comm. Pure Appl. Math. 1961. V. 14. P. 599-617.

3. Calderon A. P. Uniqueness in Cauchy problem for partial differential equations // Amer. J. Math. 1958. V. 80. P. 16-36. 
4. Ниренберг Л. Лекции о линейных дифференциалњных уравнениях с частными производными // УМН. 1975. Т. 30. № 4. С. 147-204.

5. Трев $\Phi$. Введение в теорию псевдодиффференциальных операторов и интегральных операторов Фурье. Т. 1. М.: Мир, 1984.

6. Егоров Ю. В. Линейные дифференциальные уравнения главного типа. М.: Наука, 1984.

7. Бесов О. В., Ильин В. П., Никольский С. М. Интегральные представления функций и теоремы вложения. М.: Наука, 1996.

8. Lascar $R$. Propagation des singularités des solutions d'équations pseudo-différentielles quasi homogènes // Ann. Inst. Fourier (Grenoble). 1977. V. 27. P. 79-123.

9. Sakurai T. Propagation of regularities of solutions to semilinear partial differential equations of quasi-homogeneous type // J. Fac. Sci. Univ. Tokyo Sect. IA Math. 1986. V. 33. P. 347-378.

10. Kumano-go H. Pseudo-differential operators. London: MIT Press, 1984.

11. Эйдельман С. Д. Параболические системы. М.: Наука, 1964.

12. Ландис E. M., Олейник O. А. Обобщенная аналитичность и некоторые связанные с ней свойства решений эллиптических и параболических уравнений // УМН. 1974. Т. 29. № 2. C. $190-206$.

13. Колмогоров А.Н., Петровский И. Г., Пискунов И.С. Исследование уравнения дифофузии, соединенной с возрастанием вещества, и его применения к одной биологической проблеме // Бюл. МГУ. Математика и механика. 1937. Т. 1. №6. С. 1-26.

14. Зельдович Я.Б. К теории распространения пламени горения // Журн. физ. химии. 1948. T. 22. № 1. C. 27-48.

15. Кадомиев Б. Б., Петвиашвили В.И. Об устойчивости уединенных волн в среде со слабой дисперсией // Докл. АН СССР. 1970. Т. 192. №4. С. 753-756.

Российский университет дружбы народов,

Поступила в редакцию

г. Москва

20.01.1999 и 18.08 .1999 